$50 / 20925(2)$

PPPL-2852

UC-420,427
PREPARED FOR THE U.S. DEPARTMENT OF ENERGY, UNDER CONTRACT DE-AC02-76-CHO-3073

EXCITATION OF HIGH-n TOROIDICITY-INDUCED SHEAR ALFVÉN EIGENMODES BY ENERGETIC PARTICLES AND FUSION ALPHA PARTICLES IN TOKAMAKS

BY

G.Y. FU AND C.Z. CHENG

July, 1992

manReE TON

plabma phrence

LAREIA Tolin

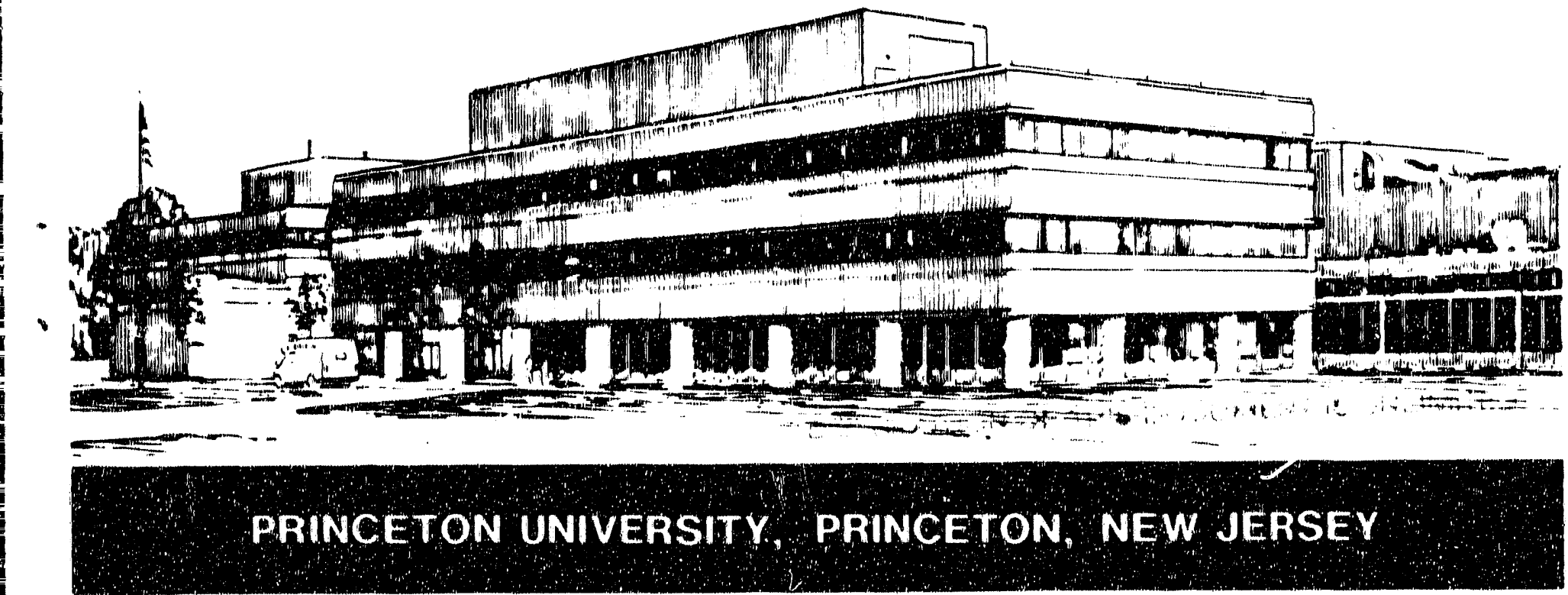




\section{NOTICE}

This report was prepared as an account of work sponsored by an agency of the United States Government. Neither the United States Government nor any agency thereof, nor any of their employees, makes any warranty, express or implied, or assumes any legal liability or responsibility for the accuracy, completeness, or usefulness of any information, apparatus, product, or process disclosed, or represents that its use would not infringe privately owned rights. Reference herein to any specific commercial produce, process, or service by trade name, trademark, manufacturer, or otherwise, does not necessarily constifute or imply its endorsement, recommendation, or favoring by the United States Government or any agency thereof. The views and opinions of authors expressed herein do not necessarily state or reflect those of the United States Government or any agency thereof.

\section{NOTICE}

This report has been reproduced directly from the best available copy.

Available to DOE and DOE contractors from the:

Office of Scientific and Technical Information

$$
\text { P.O. Box } 62
$$

Oak Ridge, TN 37831 ;

Prices available from (615) 576.8401 .

Available to the public from the:

National Technical Information Service

U.S. Department of Commerce

5285 Port Royal Road

Springfield, Virginia 22161

$703 \cdot 487-4650$ 


\title{
Excitation of high-n toroidicity-induced shear Alfvén eigenmodes by energetic particles and fusion alpha particles in tokamaks
}

\author{
C.Y. FI and C. Z. Cheng \\ Princeton Plasma Physics Laboratory \\ Princeton [nicersity \\ Princeton. .V.J. 0.954 .3
}

PPPL --2852

DE92. 016804

The stability of high-n toroidicity-induced shear Alfvén eigenmodes (TAE) in the presence of fusion alpha particles or energetic ions in tokamaks is investi. gated. The TAE modes are discrete in nature and thus can easily tap the free energy associated with energetic particle pressure gradient through wave particle resonant interaction. A quadratic form is derived for the high-n TAE modes 11sing gyro-kinetic equation. The kinetic effects of energetic particles are calculated perturbatively using the ideal MHD solution as the lowest order eigenfunction. The finite Larmor radius (FLR) effects and the finite drift orbit width (FDVI) ef. fects are included for both circulating and trapped energetic particles. It is shown that. for circulating particles. FI.R and FDW effects have two opposite influences on the stability of the high-n T.AE modes. First. they have the usual stabiliz. ing effects by reducing the wave particle interaction strength. Second. they also have destabilizing effects by allowing more particles to resonate with the T.AE motes. It is found that the growth rate induced by the circulating alpha particles

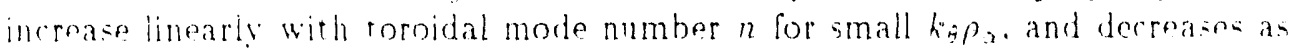
1 in for ki, , $>1$. The maximum growth rate is obtained at kap, on the order of unity and is nearly constant for the range of $0 . \bar{T}<1, \mu_{1}<2.5$. On the or her hand. the trapped particle response is dominated by the precessional drift reoronance. The bounce resonant contribution is negligible. The growth rate poaks sharply at the value of $k_{\theta} \rho_{a}$ such that the precessional drift resonance occurs for the most energetic trapped particles. The maximum growth rate due to the energetic trapped particles is comparable to that of circulating particles. Finally: the affect of the two dimensional wave structure of TAE modes is considered by using the $1 \mathrm{KKB}$ method. 


\section{INTRODUCTION}

As we are closer to the realization of tokamak plasma fusion ignition, it is extremely important to understand the novel behaviors of burning plasma associated with fusion product alpha particles. One subject, which has received increasing attentions recently, is the alpha particle destabilization of shear Alfvén waves. It has been long recognized ${ }^{1,2}$ that shear Alfvén waves can be excited by tapping the free energy source associated with the energetic particle pressure gradient through parallel wave particle interaction: One particular class of shear Alfvén waves considered in this paper is the toroidicity-induced Alfvén eigenmode ${ }^{3,4}$ (TAE). Recently, interest in TAE modes has surged following theoretical predictions $s^{5-10}$ and subsequent experimental evidences ${ }^{11,12}$ of their excitation by super-Alfvénic energetic particles. The growth rate $\gamma$ of the TAE mode induced by energetic particles may be expressed in a generic local formula.

$$
\frac{\gamma}{\omega^{\prime} T A E}=q^{2} 3_{h}\left(\frac{\omega_{*}}{\omega^{\prime} T A E}-1\right) f_{r}-\frac{\gamma_{1}}{\omega^{\prime} T \cdot A E}
$$

where $\omega_{T A E}$ is the real part of the TAE eigenfrequency. $3_{h}$ is the energetic particle (alpha particle) beta value. $q$ is the tokamak safety factor. $w$ is the riamagnetic drift frequency of energetic particle, $f_{r}$ is related to the fraction of the number of resonant particles, and finally, $\gamma_{d}$ is the damping rate of TAE mode due to core plasma kinetic effects and Alfién continuum damping. ${ }^{13-16}$ From this equation. it is clear that three conditions must be satisfied in order to destabilize TAE mode. They are: 1) the energetic particle speed must be comparable to Alfren phase speed $r$ in order to allow the parallel wave-particle resonance to occur: 2) the energetic particle pressure gradient must be steep enough so that inverse Landau damping occurs. i.e. $\omega: / \omega_{A E}>1$ : and 3) the net energetic particle destabilizing contribution must overcome the background damping of the TAE mode. Recent theor $y^{5-10.13}$ and experimental evidences ${ }^{11,12}$ have shown that low-n TAE modes can be excited by neutral beam injected hot ions with large $3_{h}$ of the energetic particles. In an ignited tokamak plasma, the fusion product alpha particles have a birth relocity comparable or larger than the Alfuén phase velocity, and have a pressure profile sharply peaked at the center of the plasma. Therefore. the first and the second condition for destabilization of the TAE mode can be easily met. However the third condition is not as easily satisfied for low-n TAE mories since the alpha particle beta value $3_{x}$ is relatively low, on an order of one percent.

In this work. we consider the excitation of high-n TAE modes by energetic ions or alpha particles. High-n TAE modes are expected to be more susceptible to the destabilization than the low-n modes. Note that, in Eq. (1). the energetic particle destabilizing term is proportional to wh which is. in turn. proportional to the toroidal 
mode number $n$. Furthermore, recent work ${ }^{14,15}$ suggests that the Alfven continuum damping of the TAE modes tends to decrease with increasing mode number $n$. On the other hand, the finite Larmor radius effects (FLR) and the finite drift orbit effects (FDW) are stabilizing, and are expected to increase with the mode number. Balance of these competing effects yields an intermediate mode number $n$ for which the TAE mode is most susceptible to the energetic particle destabilization (or the smallest critical beta).

The energetic/alpha particle effects on high-n TAE modes have been studied previously. ${ }^{8,18-21}$ Chen, ${ }^{8}$ and also Biglari, Chen and Zonca, ${ }^{18}$ considered alpha particle destabilization analytically for large aspect ratio low-beta model tokamak equilibria. in the limit of zero orbit width. Spong et al. ${ }^{19}$ studied the same problem for general numerical equilibria, but for trapped particle only. The finite orbit width was also neglected. Recently, Rewoldt ${ }^{20}$ studied the alpha particle effect on high-n Alfvén modes by using a more comprehensive approach and found that an Alfvén branch can be destabilized by fusion alpha particles for the BPX. The Alfven mode had a real frequency $\omega_{r} \approx u_{A} / q R$, and maybe related to the Ellipticity-induced Alfven eigenmodes. ${ }^{22}$

Our formulation retains full FLR effects and the main FDW effects by employing the gyro-kinetic equation. ${ }^{23.24}$ We derive a quadratic form for the high-n TAE modes from the parallel and perpendicular components of the Ampere's law and from the quasineutrality condition. We assume that the TAE modes can be described by the ideal . MHD equation and their wave structure is not effected by the presence of energetic particles. Thus, we treat energetic particle effects perturbatively, We note that similar perturbative methods have been used previously ${ }^{6.10}$ in calculating the energetic particle effects on low-n TAE modes. The TAE eigenmode structure is obtained via WKB method. ${ }^{14.16}$ The lowest order WKB solution represents the fast radial variation for each poloidal mode, whereas the higher order solution describes the slow radial variation of the amplitude. which naturally includes the physics of Alfien continuum damping. ${ }^{1+15}$ We will first present the results in the local limit using the lowest order WKB solution. The non-local effects on the energetic particle destabilization will also be studied.

In Sec. II of the present paper. a perturbative formula for the growth rate induced by energetic particles is derived. Section III presents numerical and analytical results for the destabilizing effects in the radially local limit due to fusion alpha particles as well as the background damping effects due to thermal species. The effects of the non-local radial structures of the high-n TAE mode are considered in Sec. IV. Finally in Sec $V$. discussions and conclusions are given. 


\section{FORMULATION}

Here we derive a quadratic form for the high-n TAE modes with kinetic effects of energetic particle including fusion alpha particles, as well as background plasma species. We start with the standard gyro-kinetic equation ${ }^{23.24}$ in terms of WKBballooning formalism:

$$
\left(\omega-\omega_{d}+i \sigma v_{\|}^{\prime} \mathbf{b} \cdot \nabla\right) g_{j}^{\sigma}=-q_{j} \frac{\partial F_{j}}{\partial E}\left(\omega-\omega_{*}\right)\left[J_{0}\left(\phi-\sigma \frac{v_{\|}}{c} A_{\|}\right)+\frac{v_{\perp}}{c} A_{\perp} J_{1}\right]+i C\left(g_{j}\right),
$$

where $g_{j}^{\sigma}$ is the non-adiabatic portion of the perturbed distribution function

$$
f_{j}=f_{j} \exp (i S-i \omega t)
$$

and is given by

$$
f_{j}=q_{j} \phi\left(\frac{\partial F_{j}}{\partial E}+\frac{1}{B} \frac{\partial F_{j}}{\partial \mu}\right)-q_{j} A_{\|} \frac{v_{\|}}{c} \frac{\partial F_{j}}{\partial \mu}+g_{j} \exp (i L) .
$$

In Eqs. (2)-(4), $J_{l}=J_{l}\left(|\nabla S| v_{1} / \Omega\right)$ is the th order Bessel function, $S$ is the usual eikonal that describes the fast variation of the perturbed quantities perpendicular to the magnetic field line, and $C$ is the gyro-averaged pitch angle scattering collision operator ${ }^{25}$ which is given in the guiding center limit by

$$
C\left(g_{j}\right)=\nu \frac{v^{\prime} \|}{B} \frac{\partial}{\partial \mu}\left[v_{\| \mu} \mu \frac{\partial g_{j}}{\partial \mu}\right] .
$$

More definitions in Eqs. (2)-(t) are as follows. $A_{\|}$and $A_{\perp}$ is the parallel and perpendicular component of the perturbed magnetic vector potential, respectively. $o$ is the perturbed electrostatic potential, $\omega$ is the wave frequency, $b$ is the unit vector of the equilibrium magnetic field, the subscript $j$ denotes the particle species. $F$, is the equilibrium particle distribution function, $\sigma$ is the sign of the parallel velocity ${ }^{\prime} \|, E$ is the particle energy, $\mu=m v_{\perp}^{2} / 2 B$ is the magnetic moment, $\Omega$ is the cyclotron frequency, $c$ is the speed of light, $L=\mathrm{b} \times \nabla S \cdot \mathrm{v} / \Omega$ and $\nu$ is particle collision frequency. Finally. we list the definitions for the vector field $\mathbf{A}$, the magnetic drift frequency $\omega_{d}$ and the diamagnetic drift frequency $\omega_{*}$ :

$$
\begin{aligned}
\mathrm{A} & =A_{\|} \mathrm{b}-i \frac{\mathrm{b} \times \nabla S}{|\nabla S|} A_{\perp} \\
\omega_{d} & =-\mathrm{b} \times \nabla S \cdot \frac{m v^{\prime} \|^{2} \kappa+\mu \nabla B}{m \Omega} \\
\omega_{*} & =\frac{\mathrm{b} \times \nabla S \cdot \nabla F}{m \Omega \partial F / \partial E}
\end{aligned}
$$


where $\kappa=\mathrm{b} \cdot \nabla \mathrm{b}$ is the magnetic curvature. After substituting $A_{\|} \equiv \frac{c}{i \omega} \mathrm{b} \cdot \nabla \Phi$,

$$
g_{j} \equiv-q_{j} \frac{\partial F_{j}}{\partial E}\left(1-\frac{\omega_{*}}{\omega}\right) J_{0} \Phi+h_{j}
$$

and the $b \times \nabla s$ component of the Ampere's law

$$
B_{\|}=\frac{4 \pi}{\omega B^{2}} \mathbf{b} \times \nabla S \cdot \nabla P-B_{1}
$$

into Eq. (2), we obtain a convenient form of the gyro-kinetic equation as follows:

$$
\left(\omega-\omega_{d}^{\prime}+i \sigma v^{\prime} \mid \mathbf{b} \cdot \nabla\right) h_{j}^{\sigma}=-q_{j} \frac{\partial F_{j}^{\prime}}{\partial E}\left(1-\frac{\omega_{-}}{\omega}\right) H^{\sigma}
$$

with $H_{j}^{\sigma}$ being defined as

$$
H^{\sigma} \equiv J_{0}\left(\omega \Psi+\omega_{k} \Phi\right)-J_{2} \omega_{p}^{\prime} \Phi-\frac{\omega^{\prime} \prime_{1}^{2}}{2 \Omega c}\left(J_{0}+J_{2}\right) B_{1}
$$

where we have made the following definitions:

$$
\begin{aligned}
\Psi & =\phi-\Phi \\
\omega_{p}^{\prime} & =\frac{4 \pi \mu}{q B} \mathrm{~b} \times \nabla S \cdot \nabla P \\
\omega^{\prime} k & =\omega_{d t}-\omega_{p}^{\prime} \\
B_{1} & =\frac{4 \pi}{c} \sum_{j} q_{j} \int d^{3} v \frac{v_{1}^{2}}{2 \Omega}\left(J_{0}+J_{2}\right) h_{j} .
\end{aligned}
$$

We can now proceed to derive the system of eigenmode equations from Eq. (11). the Ampere's law and the quasineutrality condition. After multiplying Eq. (11) with $J_{0} q_{j}$ on both sides, integrating over velocity space and summing over all species. We obtain

$$
\begin{aligned}
& \frac{c^{2}}{4 \pi \omega} \mathbf{B} \cdot \nabla \frac{|\nabla S|^{2}}{B^{2}} \mathbf{B} \cdot \nabla \Phi+\sum_{j} \int d^{3} v \omega\left(1-J_{0}^{2}\right) \frac{q_{j}^{2}}{T_{j}} F_{j} \Phi+\sum_{j} \omega \frac{n_{j} q_{j}^{2}}{T_{j}} \Psi \\
= & -\sum_{j} \int d^{3} v q_{j}^{2} \frac{\partial F_{j}}{\partial E}\left(1-\frac{\omega_{-}}{\omega}\right) J_{0} H^{\sigma}+\sum_{j} \int d^{3} \imath^{\prime} q_{j}\left(J_{0} \omega_{d}+i \sigma v^{\prime} \| \mathbf{b} \cdot \Gamma \cdot J_{0}\right) h_{j}^{\sigma},
\end{aligned}
$$

where we have used the parallel component of the Ampere's law

$$
\sum_{j} \int d^{3} v q_{j} v^{2}|| h_{j}=-\frac{i c^{2}}{4 \pi \omega}|\nabla S|^{2} \mathrm{~b} \cdot \Gamma \Phi
$$


and the quasineutrality condition

$$
\sum_{j} \int d^{3} v q_{j} J_{0} h_{j}=\sum_{j} \frac{n_{j} q_{j}^{2}}{T_{j}} \Psi+\sum_{j} \int d^{3} v\left(1-J_{0}^{2}\right) \frac{q_{j}^{2}}{T_{j}} F_{j} \Phi .
$$

We then expand the Bessel function $J_{0}$ in the second term of Eq. (17), by assuming small gyro-radius ordering for thermal ions. Equation (17) becomes

$$
\begin{aligned}
& \mathbf{B} \cdot \nabla \frac{|\nabla S|^{2}}{B^{2}} \mathbf{B} \cdot \nabla \Phi+\frac{\omega^{2}}{v_{A}^{2}}|\nabla S|^{2}(\Phi+\Psi)-\frac{8 \pi}{B^{2}}[\mathbf{b} \times \nabla S \cdot \nabla P][\mathbf{b} \times \nabla S \cdot \kappa] \Phi \\
= & \sum_{j} \frac{4 \pi \omega}{c^{2}} \int d^{3} v q_{j}\left(J_{0} \omega_{k}-J_{2} \omega_{p}+i \sigma v^{\prime} \| \mathbf{b} \cdot \nabla J_{0}\right) h_{j}^{\sigma} .
\end{aligned}
$$

Equations (11), (16), (18), (19) and (20) constitute our system of eigenmode equations - for four unknown fields $h_{j}^{p}, \Phi, \Psi$ and $B_{1}$.

We now derive a quadratic form from this set of eigenmode equations in order to facilitate our perturbative cillulation of the TAE growth rate induced by energetic particles. To do this, we multiply Eq. (16) with $\omega^{2} B_{1}^{*} / c^{2}$. Eq. (19) with $4 \pi \omega^{2} \Psi^{*} / 1^{2}$ and $E_{q}$. (20) with $\Phi^{*}$. add them together and integrate along the field line. After integration by part, we obtain

$$
\int \frac{d l}{B} Q\left(\Phi, \Psi, B_{1}\right)=0
$$

where

$$
\begin{aligned}
Q\left(\Phi, \Psi, B_{1}\right)= & |\nabla S|^{2}|\mathrm{~b} \cdot \nabla \Psi|^{2}+\frac{S_{j}}{B^{2}}[\mathrm{~b} \times \nabla S \cdot \Gamma P][\mathrm{b} \times \nabla S \cdot \kappa]|\Phi|^{2} \\
& -\omega^{2} \frac{|\nabla S|^{2}}{l^{2}}|\Phi|^{2}-\omega^{2} \frac{\mid \nabla S^{2}}{l^{2}}\left(\Phi^{*} \Psi+\Phi \Psi^{*}\right)+\frac{\omega^{2}}{c^{2}}\left|B_{1}\right|^{2} \\
& -\sum_{j} 4 \pi \frac{\omega^{2}}{c^{2}} \frac{n, q_{j}^{2}}{T_{j}^{2}}|\Psi|^{2}+\sum_{j} 4 \pi \frac{\omega}{c^{2}} \int d^{3} r q_{j}\left(H^{\sigma}\right)^{*} h_{j} .
\end{aligned}
$$

Our derivation is similar to that of $\mathrm{Xu}$ and Rosenbluth, ${ }^{26}$ but with one important difference that we retain full FLR effects. Note the first three terms in Er (??) constitute the ideal MHD equation without compression effect: (i.e.. $5_{1}$ term) and kinetic effects (i.e., the parallel electric field term $\Psi$ and the non adiabatic $h$, ). The growth rate due to kinetic effects can now be calculated perturbatively. Let $\omega=$ $\omega_{0}+\delta \omega, \Phi=\Phi_{0}+\delta \Phi$ and drop small $\Psi$ terms and $B_{1}$ terms momentarily, where $\omega_{0}$ and $\Phi_{0}$ denotes the ideal eigenfrequency and eigenfunction, respectively; the $\delta$ terms are the corresponding kinetic corrections assumed to be small. Ising this ordering. 
the quadratic form can be expanded and the lowest order terms [the first three terms in Eq. (22)] yields the following ideal $\mathrm{MHD}$ equation for $\Phi_{0}$ :

$$
\mathbf{B} \cdot \nabla \frac{|\nabla S|^{2}}{B^{2}} \mathbf{B} \cdot \nabla \Phi_{0}+\frac{\omega^{2}}{v_{A}^{2}}|\nabla S|^{2} \Phi_{0}-\frac{8 \pi}{B^{2}}[\mathbf{b} \times \nabla S \cdot \nabla P][\mathbf{b} \times \nabla S \cdot \kappa] \Phi_{0}=0 .
$$

The next order equation determines $\delta \omega$ which is given by

$$
\frac{\delta \omega}{\omega}=\frac{\sum_{j} N_{j}}{W}
$$

where

$$
\begin{aligned}
& V_{j}=\frac{2 \pi}{\omega_{0} c^{2}} \int \frac{d l}{B} \int d^{3} v q_{j},\left(H^{\sigma}\right)^{*} h_{j} \\
& W=\int \frac{d l}{B} \frac{|\nabla S|^{2}}{v_{A}^{2}} \Phi_{0}^{2} .
\end{aligned}
$$

Here $V_{j}$ represents the kinetic contribution due to particle species $j$, which includes energetic particle, as well as thermal electrons and ions. Note that the solution $h_{j}^{\circ}$ is still unknown at this point. We $\mathrm{n}$ solve $\mathrm{Eq}$. (11) for $h_{j}^{\sigma}$. The solution for the circulating particles is

$$
h_{j}^{\omega}=q_{j} \frac{\partial F_{j}}{\partial E}\left(1-\frac{\omega_{k}}{\omega}\right) \int_{-\infty}^{+\infty} d k \frac{H^{\sigma}(k) \exp \left(i k \theta_{c}-i \sigma I_{c}(\theta)\right)}{\sigma k \omega_{c}-\omega} .
$$

where

$$
\begin{aligned}
H^{\sigma}(k) & =\int_{-\infty}^{+\infty} d \theta_{c} \exp \left(-i k \theta_{c}+i \sigma I_{c}(\theta)\right) H^{\sigma}(\theta), \\
\theta_{c} & =\omega_{c} \int_{0}^{\theta} \frac{J B}{v_{\|}} d \theta . \\
I_{c}(\theta) & =\int_{0}^{\theta} \omega_{t} \frac{J B}{v^{\prime} \|} d \theta .
\end{aligned}
$$

and $\omega_{c}$ is the transit frequency of the circulating particles. The solution for trapped particles is

$$
h_{j}^{\sigma}=\frac{V_{j}^{+}\left(\theta_{m}\right)+V_{j}^{-}\left(\theta_{m}\right)}{2 \sin \left(\bar{I}_{b}\left(\theta_{m}\right)\right)} \exp \left[i \sigma \bar{I}_{b}(\theta)\right]-i \sigma V_{j}^{\sigma}(\theta),
$$

where

$$
\begin{aligned}
V_{j}^{\prime}(\theta) & =\int_{\frac{-\pi}{2}}^{\theta_{b}} d \theta_{b}^{\prime} H^{\sigma} \exp \left(-i \sigma\left(\bar{I}_{b}^{\theta^{\prime}}-\bar{I}_{b}^{\theta}\right)\right), \\
\tilde{I}_{b}(\theta) & =\int_{-\theta_{m}}^{\theta} \frac{J B}{v^{\prime} \|} d \theta\left(\omega-\omega_{d}\right), \\
\theta_{b} & =\omega_{b}^{\prime} \int_{-\theta_{m}}^{\theta} \frac{J B}{v^{\prime} \|} d \theta^{\prime}-\frac{\pi}{2} .
\end{aligned}
$$


Here, $\omega_{b}$ is the bounce frequency of the trapped particles, $\theta_{m}$ is the poloidal angle corresponding to the turning point of the banana orbit, $\theta_{b}$ is the bounce angle and $J$ is the coodinate transform Jacobian. Finally, we list a useful formula to be used in calculating the resonant contribution of the trapped particles:

$$
\begin{aligned}
\operatorname{Res}\left[\oint d \theta_{b}\left(H^{\sigma}\right)^{*} h_{j}^{\sigma}\right] & =\frac{2}{\omega_{b}} \cot \left(\bar{I}_{b}\left(\theta_{m}\right)\right) \mid \oint d \theta_{b}\left[H_{1} \cos \left(\bar{I}_{b}(\theta)\right)+\left.H_{2} \sin \left(\vec{I}_{b}(\theta)\right]\right|^{2}\right. \\
& =-2 i \sum_{p}\left|H_{p}^{l}\right|^{2} \delta\left(\omega-p \omega_{b}-\bar{\omega}_{d}\right)
\end{aligned}
$$

where Res denotes the resonant part of the contribution and

$$
\begin{aligned}
H_{p}^{l} & =\oint d \theta_{b}\left[H_{1}(\theta) \cos \left(p \theta_{b}-I_{b}(\theta)\right)+H_{2}(\theta) \sin \left(p \theta_{b}-I_{b}(\theta)\right)\right] \\
I_{b}(\theta) & =\int_{-\theta_{m}}^{\theta}\left(\omega_{i l}-\bar{\omega}_{t l}\right) \frac{J B}{v_{\|}} d \theta .
\end{aligned}
$$

Here the bounce integration for $H_{p}^{l}$ is done at the th trapped particle region. namely $2 \pi l-\theta_{m}<\theta<2 \pi l+\theta_{m}$.

After plugging $h^{\sigma}$, into $E_{q}$. (2.t) for both circulating particles and trappert particles. the growth rate $\gamma=-i \delta \omega$ can be determined straightforwardly and is given by

$$
\frac{\gamma}{\omega^{\prime} 0}=\sum_{j} \frac{2 \pi^{2} \int E d E\left(-q_{j}^{2} \partial F / \partial E\right)\left(\omega_{*} / \omega_{0}-1\right)\left(N_{j}^{u}+N_{j}^{t}\right)}{m_{j}^{2} c^{2} B^{2} \omega_{0} \int_{-\infty}^{+\infty}\left(|\nabla S|^{2} \Phi_{0}^{2} J / \nu_{j}^{2}\right) d \theta}
$$

where $J$ is the coodinate transform Jacobian, $V_{j}^{u}$ and $V_{j}^{t}$ represents the contribution due to circulating particles and trapped particles, respectively, and

$$
\begin{aligned}
& V_{j}^{u}=\int_{1}^{\lambda_{1}} d \Lambda \int_{-\infty}^{+\infty} d k \frac{1}{\omega_{c}}\left(\left|H^{+}(k)\right|^{2}+\mid\left(.\left.H^{-}\left(-k^{\prime}\right)\right|^{2}\right) \delta\left(k \omega_{c}-\omega^{\prime}\right)\right. \\
& V_{j}^{t}=\int_{\lambda_{1}}^{\lambda_{2}} d \Lambda \sum_{l_{1, p}=-\infty}^{+\infty} \frac{1}{\omega_{b}}\left|H_{p}^{l}\right|^{2} \delta\left(p \omega_{b}+\bar{\omega}_{d}-\omega\right) .
\end{aligned}
$$

In Eq. (39) and $(40), H^{ \pm}(\mp k)$ is the Fourier transiormation of $H^{\sigma}(\theta)$ for circulating particles, $\bar{\omega}_{d}$ is the bounce averaged magnetic drift frequency, and $. l=\mu B_{0} / E$ is the particle velocity pitch angie variable with $\Lambda_{1}$ corresponding to the trapped and circulating boundary and $I_{2}$ corresponding to the deeply trapped particles.

\section{NUMERICAL AND ANAIYTICAL RESULTS: LOCAL THEORY}

In this section, we evaluate the growth rate of the high-n TAE mode due to energetic particles and the damping due to thermal electrons and ions in the local limit. By 
the local limit, we mean that the eigenfunction $\Phi$ is approximated by the lowest order WKB solution. In general, the WKB solution can be written as

$$
\Phi=A(r) \Phi(r, \theta) \exp (i n S)
$$

where $S=\zeta-q(r) \theta$ is the WKB eikonal, $\theta$ and $\zeta$ is the poloidal and toroidal angle respectively, $r$ is the magnetic flux variable and is a measure of the mean radius. In Eq. (41), exp (inS) describes the fast variation of the TAE mode across the field line, $\Phi(r, \theta)$ describes the slow variation along the field line and is determined by the lowest order WKB equation; whereas $A\left(r^{\circ}\right)$ describes the slow variation of the radial envelope for these poloidal modes, and is determined by the higher order WKB equation. ${ }^{16}$ In the lowest order, $A(r)$ is constant. In this section, we calculate the growth rate according Eq. (38) in this local limit. The results with correction due to the non-local higher order terms will be presented in the next section.

Although our formulation is valid for general non-circular tokamak equilibria. We consider large aspect ratio. low-beta model equilibria ${ }^{27}$ with shifted circular magnetic surfaces in order to simplify our problem. For this model equilibrium. the ideal MHHD equation Eq. (23) becomes the well known high-n ballooning mode equation:27

$$
\begin{aligned}
& \frac{\partial}{\partial \theta}\left[G(\theta)\left[1+h^{2}(\theta)\right] \frac{\partial}{\partial \theta} \Phi+\bar{\omega}^{2} G^{\prime}(\theta)(1+4 \epsilon \cos \theta)\left[1+h^{2}(\theta)\right] \Phi\right. \\
& +\Delta_{F}[\cos \theta+h(\theta) \sin \theta] \Phi=0,
\end{aligned}
$$

where $h(\theta)=s\left(\theta-\theta_{k}\right)-\Delta_{p} \sin \theta \cdot C r(\theta)=1+2 \Delta^{\prime} \cos \theta, s=q d q / q d r . \Delta_{p}=$ $-2 q^{2} R(d P / d r) / B^{2}, \Delta^{\prime}$ is the radial derivative of the Shafranov shift. $\epsilon=r / R$. and $\bar{\omega}=\omega / \omega_{A}$ with $\omega_{A}=v_{A} / q R$ being the Alfven frequency. Finally $\theta_{k}$ is the radial wave number which describes the slow radial variation. For our local limit assumed here. $\theta_{k}$ is chosen to be zero.

In this work. we mainly consider fusion alpha particles. The particle distribution function is described by a slowing-down energy distribution with uniform pitch angle. We will consider the contributions from circulating particles and trapped particles separately. The key parameters are $v_{\alpha} / v_{A}$ (the ratio of alpha birth speed to Alfvén phase velocity) and $k_{\theta} \rho_{\alpha}$ (the finite orbit width parameter), where the Larmor radius is defined with the hirth alpha velocity. Finally, we model the alpha particle density profile as $n_{\alpha}(r)=n_{\alpha}(0) \exp \left(-\left(r / L_{\alpha}\right)^{2}\right)$ where $L_{\alpha}$ is the density scale length. To obtain the alpha particle-induced growth rate, we first obtain the numerical solutions of Eq. (42) for $\Phi$ by shooting method. The boundary conditions are $\Phi( \pm \infty)=0$. After we obtain $\Phi$, we plug it into Eq. (38) to calculate the growth rate perturbatively. For circulating particle contribution, we perform numerical integration in $\theta, k$, and .1 : the energy integration can be done analytically due to resonant $\delta$ function. For the 
trapped particle contribution, we first carry out integration in $\theta_{b}$ for the 1 th trapped region, then sum up $l$ and the bounce harmonic $p$, and finally carry out the pitch angle integration.

In Subsection A, we will calculate the destabilizing contribution of the circulating alpha particles, while in Subsection B, we will consider the destabilizing contribution of the trapped alpha particles. Subsection $\mathrm{C}$ is devoted to the damping rutes of the TAE mode due to thermal particle species. Finally, in Subsection D, we evaluate the critical beam beta value for the excitation of the TAE mode in the TFTR NBI experiment. ${ }^{11}$

\section{A. Circulating Particle Contribution}

We first consider the destabilizing contribution of circulating alpha particles. The parameters of $\epsilon=0.1, q=1.0, L_{p} / R=0.1$ and $\beta_{\alpha}(0)=2 \%$ are chosen. Figure 1 shows the growth rates induced by circulating alpha particles as a function of $v_{\alpha} / v_{A}$ for parameters of $s=0.6$ and $\Delta_{p}=0.0$ and $k_{\theta} \rho_{a}=1.0$. The dashed curve is obtained in the limit of zero orbit width [i.e., the FLR and the FDVV effects are turned off in Eq. (39), but $\omega_{\alpha} \times k_{\theta} \rho_{\alpha}$ is kept finite]. The solid curve corresponds to the results with finite orbit width effects. We see that the finite orbit effects are stabilizing for $v_{\alpha} / v_{A}>1.1$ and destabilizing for $v_{\alpha} / v_{A}<1.1$. This exhibits two opposite influences of finite orbit size. On one hand, finite orbit width has a stabilizing effect by reducing the wave-particle interaction strength, on the other, this orbit width effect has a destabilizing effect by bringing more particles into resonance with the waves. Figure 2 shows function $\mathrm{H}^{+}(k)$ with and without orbit width effects at the resonant energy: Physically, $H(k)$ is related to the work done on the resorant particles by the perturbed alectrical field. and $k$ is related to the radial variable $n q-m$ for the poloidal mode number $m$. Thus, $H(k)$ is a measure of the strength of the wave particle interaction as a function of the radius. Figure 2 shows that, in the limit of zero orbit width, $H(k)$ peaks sharply at $k=1 / 2$ and $k=3 / 2$, reflecting the fact that the TAE morle peaks at $n q-m= \pm 1 / 2$ magnetic surfaces with a radial localization width on the order of $\epsilon r / n$. However, with finite orbit width effects, $H(k)$ is shown to have a much broader peak with smaller amplitude near $k=1 / 2$ (Note that an additional peak appears at $k=5 / 2$ due to the poloidal mode coupling induced by the magnetic drift orbit). Physically, these broader peaks mean that particles away from where mode localizes can still interact effectively with the mode due to the finite orbit width effects. It is clear that the destabilizing effect of the finite orbit width is a direct consequence of the radially localized structure of the TAE mode.

We now study the variation of the growth rate as a function of $k_{\theta} \rho_{\alpha}$. It is known that without orbit width effects, the growth rate is a linear function of $k_{\theta} \rho_{x}$. However 
this linear dependence is expected to be modified by the finite orbit width. Figure 3 shows the growth rate as a function. of $k_{\theta} \rho_{\alpha}$ with orbit width effects for parameters of $s=0.6 . \Delta_{p}=0$ and $v_{\alpha} / v_{\alpha}=2.0$. We observe that, as $k_{\theta} \rho_{\alpha}$ increases, the growth rate first increases linearly, then saturates at $k_{\theta} \rho_{\alpha}=0.8$, and finally decreases for $k_{\theta} \rho_{\alpha}>1.0$. Note that the value of $k_{\theta} \rho_{\alpha}$ which maximizes the growth rate is on an order of unity. Naively, one would expect that $\left(k_{r} \rho_{\alpha}\right)_{\max }$ is on the order of unity and $\left(k_{\theta} \rho_{\alpha}\right)_{\max }$ is on the order of $\epsilon=r / R$. We note that our numerical results differs significantly from that of Ref. 21. First, our results show that the growth rate is maximized at $k_{\theta} \rho_{c r}$ on an order of unity instead of $k_{\theta} \rho_{c x} \ll 1$ as implied in Ref. 21. Second. we have shown that the growth rate eventually decreases as a function of $k_{\theta} \rho_{a}$ for $k_{A} \rho_{a} \gg 1$ whereas the results of Ref. 21 imply a constant growth rate for $k_{\theta} \rho_{\alpha} \gg \Gamma$. Next we consider the variation of $\left(k_{\theta} \rho_{\alpha}\right) l_{\max }$ and the corresponding $\left(\gamma_{\alpha} / \omega_{s}\right)_{\max }$, with $v_{\alpha} / v_{A}$, as shown in Fig. 4 , for parameters of $s=0.6$ and $\Delta_{p}=0$. We see that $\left(k_{\theta} \rho_{\alpha x}\right\}_{\max }$ decrease as $v_{\alpha} / v_{A}$ increases. and $\left(i_{\alpha} / \omega_{4}\right)_{\max }$ is nearly constant in the range of $0.7<v_{\alpha} / r_{A}<2.0$.

Vext, we study the effects of the magnetic shear $s$ and the curvature pressuregradient parameter $\Delta_{p}$ on TAE stability. For $s=0.6$ and $\Delta_{p}=0$, the high-n TAE mode frequency is about at the center of the Alfren continum gap." which corresponds to the most global radial wave structure. As $s$ and $\lambda_{p}$ varies. the eigenfrequenc: shifts towards either the bottom or the upper edge of the continuum gap and its eigenfunction becomes more localized in the radial space ${ }^{28}$ (or more extended in the Fourier $\theta$ space). Figure 5 shows $\left(k_{\theta} \rho_{\alpha}\right)_{\max }$ and $\left(\gamma_{\alpha} / \omega_{4} i_{\max }\right.$ as a function of $s$ for $\lrcorner_{p}=0$. First. it is evident that $\left(k_{g} \rho_{x}\right)_{\max }$ is not sensitive to the magnetic shear. second. the maximum growth rate peaks at $s=0.6$. It has been shown ${ }^{3}$ for the $J_{F}=0$ case that as the magnetic shear decreases (increases) from $s=0.6$. the mode frequency decreases (increases) towards the bottom (top) edge of the cont inum gap and the eigenfuntion becomes increasingly localizer in the real space. Physically. Fig. 5 indicates that the growth rate becomes smaller as the mode becomes more

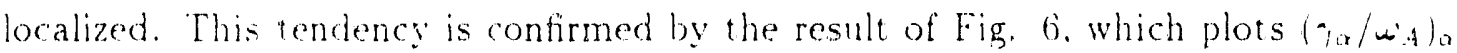
and $\left(k_{\theta} \rho_{a}\right)_{\max }$ as a function of $\Delta_{p}$ at $s=0.6$. We see that the growth rate decreases as $\lambda_{p}$ increases. whereas $\left(k_{\theta} \rho_{\alpha}\right)_{\max }$ is nearly constant. This is related to the fact 28 that the TAE eigenfrequency shifts downward to the bottom edge of the continuum gap as $\Delta_{p}$ increases. In particular. the TAE mode merges into the continum and becomes singular when $\Delta_{p}>\left(\Delta_{p}\right)_{\text {art }}=0.225$. where $\left(\Delta_{p}\right)_{\text {erit }}$ is the critical value for the existence of discrete TAE mode. It can be analytically shown that the growth rate tends to zero as $\lambda_{p}$ approaches $\left(\Delta_{p}\right)_{\text {irn }}$.

The numerical results shown above for the circulating particles can be understood analytically in certain asymptotic limits. To simplify our analytic derivation. we 
normalize function $H(\theta)$ into a dimensionless form such that

$$
\tilde{H}(\theta)=\frac{q B R}{c E k_{\theta}} H(\theta)
$$

The function $H(k)$ and $H_{p}^{l}$ is normalized in the same way to $\bar{H}(k)$ and $\vec{H}_{p}^{l}$. respectively. To make analytic progress, we consider the case of zero pitch angle. Also we use an asymptotic form ${ }^{3.28}$ to approximate the high-n TAE solution:

$$
\Phi_{0}(\theta)=\frac{\cos (\theta / 2)+\lambda \sin (\theta / 2)}{\sqrt{1+(s \theta-\alpha \sin \theta)^{2}}} \exp (-\Gamma \theta) .
$$

where $\lambda=\Omega_{+} / \Omega_{-} \sim O(1), \Gamma=\Omega_{+} \Omega_{-} \sim O(\epsilon)$, and $\Omega_{ \pm}= \pm\left[\bar{\omega}^{2}(1+\bar{\epsilon})-1 / t\right]$ with $\bar{\epsilon}=2.5 \epsilon$ in the low beta limit. We can then compute $\bar{H}(k)$ in the limit of zero orbit size, and obtain

$$
H(k) \approx \frac{\lambda \Gamma+\frac{1}{2}-k}{\left(\frac{1}{2}-k\right)^{2}+\Gamma^{2}}+\frac{-\lambda \Gamma+\frac{3}{2}-k}{\left(\frac{3}{2}-k\right)^{2}+\Gamma^{2}},
$$

where we have made use of the fact that the secular term s $\theta$ makes a dominating contribution in the limit of $\Gamma \ll 1$. Note that $\bar{H}(k)$ peaks at $k=1 / 2$ and $k=3 / 2$ with a narrow width of $\Gamma \sim O(\epsilon)$. This analytic result agrees with the mumerical result shown in Fig. 2 (dashed curre). Using Eq. (45), the growth rate ran be written into a simple form as

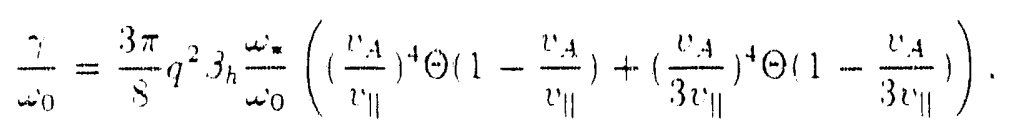

where $\theta$ is the Heaviside step function. This result is similar to the results obtained by Chen ${ }^{3}$ and by Biglari. Chen and Zonca. ${ }^{18}$

We now consider the case with finite orbit width effec*s. We note that the gyro radius is zero for zero pitch angle assumed here. but the particles can deviate from magnetic field lines due to the finite radial magnetic drift. This drift effect is contained in the secular term of the drift phase $I_{c}(\theta)$ in Eq. (30). To make analytic progress. we take the asymptotic limit $1>k_{\theta} \rho_{\alpha} \gg \Gamma$ and $I \ll 1$. In this limit, the secular term in $\bar{H}(\theta)$ dominates the contribution to the integral $\bar{H}(k)$. We may then take the asymptotic form of $I_{c}(\theta)$ for large $\theta$ and expand in Bessel functions:

$$
\exp \left(i I_{c}(\theta)\right) \approx J_{0}(z \theta)+2 \sum_{i}(-i)^{l} J_{l}(z \theta) \cos (l \theta) .
$$


where $z=\bar{v}_{\|} k_{\theta} \rho_{\alpha}$ with $\bar{v}_{\|}=v_{\|} / v_{\alpha} \leq 1$. By keeping only the principle resonance term (i.e., for $k$ near $1 / 2$ ), the integral $\bar{H}(k)$ then reduces to

$$
\bar{H}(k)=\frac{2}{z}\left(\frac{\bar{k}}{z}+\lambda \sqrt{1-\left(\frac{\bar{k}}{z}\right)^{2}}\right)
$$

for $|\bar{k}|<z$ where $\bar{k}=1 / 2-k$, and

$$
\vec{H}(k)=\frac{2}{z} \operatorname{sign}(\bar{k})\left(\frac{|\vec{k}|}{z}-\sqrt{\left(\frac{\vec{k}}{z}\right)^{2}-1}\right)
$$

for $|\bar{k}|>z$. Equations $(48)$ and $(49)$ show that the peak of $\ddot{H}(k)$ at $k=1 / 2$ now has a width on an order of $k_{\theta} \rho_{\alpha} \gg \Gamma$. Thus the width of the peak is broaden by the finite orbit width effects. This result agrees with the numerical result shown in Fig. 2. In the limit case of $1 \gg k_{0} \rho_{\alpha} \gg \Gamma$. the $k$ integration in $E_{q}$. (38) can be carried out analytically and the resulting growth rate is independent of tapa or the mode number. "However. for finite $k_{\theta} p_{\alpha}$ on order of unity the $k$ integration has to be done numerically due to the energy dependence in $z$ and $H(k)$. Our numerical result in Fig. 3 indicates that. as $k_{\theta} \rho_{a}$ increases from zero. the growth rate first increases linearly as a function of $k_{\theta} \rho_{\alpha}$ for small $k_{\theta} \rho_{\alpha}$, then it reaches its maximum at $k_{\theta} \rho_{\alpha}$ on the order of unity and finally decreases for $k_{\theta} \rho_{\alpha}>1$. The decrease in the growth rate for $k_{\theta} \rho_{a} \gg 1$ can be understood analytically in the following way. After integrating in energy: the growth rate given by $\mathrm{Eq} .(38)$ is proportional to the following $k$ integral $k_{\theta} \rho_{\alpha} \int_{k_{m}}^{\infty}\left(k_{m} / k\right)^{4}(H(k))^{2} d k$. Since $H(k)$ scales as $1 / k_{\theta} \rho_{\alpha}$ in the limit of $k_{\theta} \rho, \gg 1$ due to the fast drift phase $I_{1}(\theta)$, the growth rate scales as $1 / k_{a} \rho_{x}$ asymptotically for $k_{i} \rho_{a} \gg 1$.

\section{B. Trapped Particle Contribution}

Here we consider the trapped energetic particle contribution to the growth rate of the TAE mode. As in the last subsection. we study the finite orbit width effects and the dependence of the growth rate on $v_{\alpha} / v_{A}, k_{\theta} \rho_{\alpha} . s$ and $\Delta_{p}$. The same fixed parameters of $\epsilon, q, L_{p} / R$ and $\zeta_{\alpha}(0)$ are used. First, we demonstrate the finite orbit width effects. Figure $i$ shows the growth rates as a function of $r_{x} / l^{\prime}+$ with or without orbit width effects. for $s=0.6, \lambda_{p}=0$ and $k_{\theta} p_{\alpha}=1.0$. In the limit of zero orbit width. we turn off the FLR term contained in the Bessel functions and the finite banana width term contained in the drift phase term $I_{d}(\theta)$ in Eq. (40). but we still keep the w. and and terms. We see that the growth rate is always reduced by finite orbit width effect in contrast with that of circulating particles. Furthermore. there is 
sharp transition in the growth rate at $v_{\alpha} / v_{A}=1.0$. Physically the transition occurs at the precessional drift resonance $\bar{\omega}_{d} \approx \omega$. Note that the precessional drift resonance condition for deeply trapped particles is given by $k_{\theta} \rho_{\alpha} \bar{E} \approx v_{A} / v_{\alpha}$, where $\bar{E} \leq 1$ is the particle energy normalized to the birth energy of the alpha particle. Thus. for the parameterc of Fig. 7 . the precessional resonance can be satisfied for $v_{\alpha} / v_{A} \geq 1.0$. We also find that the bounce resonance contribution is much smaller than the precessional resonance contribution. This result has been confirmed for other parameters. It is also borne out by the result of Fig. 8 , which shows the normalized growth rate as a function of $k_{\theta} \rho_{\alpha}$ for parameters of $s=0.6, \Delta_{p}=0$ and $v_{\alpha} / v_{A}=2.0$. We notice from Fig. 8 that the sharp transition occurs at $k_{\theta} \rho_{\alpha} \approx 0.5$, precisely when the precessional drift resonance begins to occur. We also see that the growth rate then decreases rapidly due to the finite orbit width effect. The maximum growth rate is comparable to that of circulating particles as shown in Fig. 3, but the growth rate is much more narrowly peaked at $k_{\theta} \rho_{\alpha} \approx 0.6$.

We now study the dependence of $\left(k_{\theta} \rho_{\alpha}\right)_{\max }$ and $\left(\gamma_{\alpha} / w_{t}\right)_{\max }$ on $l_{\alpha} / l_{1}$. as shown in Fig. 9 obtained for parameters of $s=0.6$ and $\Delta_{p}=0$. We observe that the maximum growth rate increases with $v_{a} / v_{A}$ and the increase is more gradual for larger values of $v_{\alpha} / u_{1}$. On the other hand. $\left(k_{\theta} \rho_{x}\right)_{\max }$ decreases with $r_{a} / r_{A}$. which agrees with our analytic result of $\left(k p_{a}\right)_{\max }=r_{A} / v_{0}$. Finally their dependence on the magnetic shear $s$ and the curvature pressure-gradient parameter $\lrcorner_{p}$ are shown in Figs. 10 and 11. Overall results are similar to those for circulating particles.

Vext. we derive analytic results for trapped particle contribution. we will demonstrate that the bounce resonance contribution of the trapped alpha particles is much smaller than the precession resonance contribution. We first consider the limit of zero orbit width. For even bounce harmonic $p$ (including $p=0$ ). we obtain

$$
\begin{aligned}
H_{p}^{0} & \approx \cos \left(\frac{\pi}{2} p\right) \oint d \theta_{h}(\cos \theta+(s \theta-\alpha \sin \theta)) \cos \left(p\left(t_{h}\right) .\right. \\
H_{p}^{l \neq 0} & \approx \lambda \exp (-2 \pi \Gamma l) \cos \left(\frac{\pi}{2} p\right) \oint\left(d \theta_{h} \sin \theta \sin \frac{\theta}{2}:\right.
\end{aligned}
$$

while for order $p$, we obtain $\ddot{H}_{p}^{0}=0$ and

$$
\bar{H}_{p}^{(\neq 0)} \approx \exp (-2 \pi \Gamma l) \sin \left(\frac{\pi}{2} p\right) \oint d \theta_{b} \sin \theta \cos \frac{\theta}{2} \sin \left(p \omega_{i b}\right)
$$

It is then straight forward to demonstrate that $\left(\bar{H}_{0}^{0}\right)^{2} \gg 2 \sum_{l}\left(\ddot{H}_{p \neq 0}^{l}\right)^{2}$ (i.e. the precessional drift resonance contribution is much greater than the bounce contribution) for the deeply trapped particles. For finite pitch angle and very small $\Gamma$. the bounce contribution may be comparable to that of precessional drift resonance. However. 
the bounce contribution can be dramatically reduced by the effect of finite banana width. Therefore, the precession resonance dominates for all pitch angle.

We now consider the case with finite orbit width effect. To make analytic progress. we examine the limit of deeply trapped particles. In this limit, we have shown that $\bar{H}_{0}^{0} \gg \bar{H}_{0}^{l \neq 0}$, thus we may keep only the contribution from the $l=0$ trapped particle region. After some algebra, we obtain

$$
\frac{\gamma_{\alpha}}{\omega_{0}} \approx \frac{3}{2} \sqrt{\frac{\epsilon}{2}} q^{2}, 3_{\alpha} \frac{\left.\left(\omega_{0} / \tilde{\omega}_{d m}\right)^{2}\left(\omega_{-} / \omega_{0}\right)\right)-\frac{3}{2}\left(\omega_{0}^{\prime} / \bar{\omega}_{d m}\right)}{\int_{0}^{\infty} d \theta\left(1+h^{2}(\theta)\right) \Phi_{0}^{2}}\left(\bar{H}_{0}^{0}\right)^{2}
$$

for $\bar{\omega}_{d m} \geq \omega_{0}$, where $\bar{\omega}_{d m}$ is the maximum precessional drift frequency. $\vec{H}_{0}^{0}$ can be straightforwardly calculated to obtain

$$
\bar{H}_{0}^{0} \approx \pi J_{0}\left(\sqrt{k_{\theta} \rho_{\alpha} \frac{\omega_{0}^{\prime} 0}{\bar{\omega}_{i d m}^{\prime}}}\right) .
$$

After calculating the $\theta$ integration in the denominator by using the asymptotic form for the eigenfunction $\Phi_{0}$. Eq. (5.3) becomes

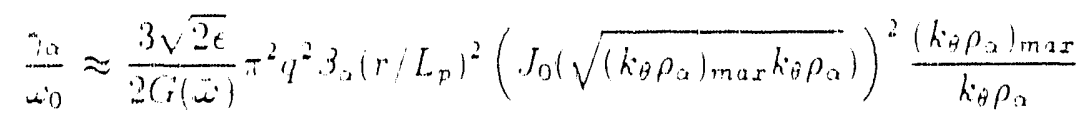

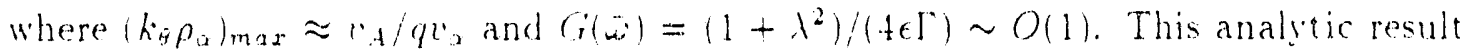
for the trapped particle contribution agrees very well with our numerical results fe.g. Fig. (s)].

\section{Damping Due To Thermal Particle Species}

Here we con ter the stabilizing effects due to thermal particle species. We first consider the dauping rate due to thermal ions. We note that the thermal speed $c$ is in general murh smaller than $v_{1}$, thus the $c^{\prime} \|=l_{4} / 3$ sideband resonance is dominating over the principle resonance. Also the finite orbit effecis may be neglected since $k_{\theta} \rho_{i} \ll 1$. C sing the results of Eq. (45). the damping rate can then be straightfor. wardly derived to obtain

$$
\frac{x_{i}}{n_{0}} \approx \sqrt{\pi} q^{2} 3,\left(1+x^{2}\right) x^{3} \exp \left(-x^{2}\right)
$$

where $x={ }^{\prime} A / 3 v_{1}$. Note that we have used the Maxwellian distribution for ions. This analytical expression agrees very well with our numerical results. We observe that the damping rate increases rapidly with ion beta due to the exponential dependence. For $q=1$. we find $\mu_{1} / w_{0} \geq 1 \%$ for $\beta_{1} \geq 2 \%$. Therefore the ion damping could be substantial for moderately high ion beta ralue. 
Next, we consider the damping rate of high-n TAE mode due to thermal electrons. It has been shown that the usual electron Landau damping is very small since thermal electron velocity is much larger than the Alfvén velocity for typical tokamak parameters. Gorelenkov and Sharapov ${ }^{29}$ showed that the dominating damping mechanism due to thermal electrons is the collisionality of trapped particles. Here, we derive the damping rate of the high-n IAE mode induced by the collisionality of trapped electrons. We start from Eq. (11) for electrons. After neglecting small electron gyroradius and substituting $\Psi$ in terms of $\Phi$ using the quasineutrality condition. Eq. (11) becomes

$$
\left(\omega-i \sigma v^{\prime} \mathbf{b} \cdot \nabla-i C\right) h_{e}^{\sigma}=q_{e} \frac{F_{e}}{T_{e}} H_{e}
$$

with

$$
H_{*}=\left(-\frac{\omega^{\mid}|\nabla S|^{2} \bar{m}_{i}}{2 \Omega_{e}^{2} m_{e}^{2}} T_{e}+\omega_{k}-\hat{\omega}_{k}\right) \Phi
$$

where $\omega_{k}$ is the electron curvature drift frequency averaged over Maxwellian distribution function. Note that in Eq. (58). the thermal ion FLR effect is kept to include the finite parallel electric field. To solve Eq. (57), we expand he in terms of small parameter $w / w h \ll 1$. The zeroth order equation reads $b . \Gamma h_{r}=0$, which implies that $h_{f 0}$ is constant along the field lines, whereas the next order terms yield

$$
\text { L - - i }<C>h_{\epsilon 0}=q_{e} \frac{F_{e}}{T_{e}}<H_{e}>
$$

where $<\rangle$ denotes the average over the banana orbit and $\langle C\rangle$ is the bounce averaged collisional operator given by 25

$$
<C>=2 \bar{\nu}\left(\frac{E}{T_{e}}\right)^{-3 / 2} \frac{Z_{\cdot f \rho}+\Pi\left(E / T_{e}\right)}{\oint \cdot 1 \theta / \sqrt{1-1 B}} \frac{\partial}{\partial .1} \cdot 1 \rho d \theta \sqrt{1-.1 B} \frac{\partial}{\partial .1}
$$

with the function $\Pi(z)$ being defined as

$$
\Pi(z)=\frac{1}{\sqrt{\pi} z} e^{-z^{2}}+\left(1-\frac{1}{2 z^{2}}\right) \frac{z}{\sqrt{\pi}} \int_{0}^{z} e^{-t^{2}} d t
$$

where $\bar{\nu}$ is a normalized electron collision frequency and is given by $\bar{\nu}=$ $4 \pi n_{\varepsilon} \epsilon^{4} \ln .1 /\left(m_{e}^{2} v_{e}^{3}\right)$. Following Rosenthluth et al. ${ }^{25}$ we solve Eq. (59) in the limit of $\bar{\nu} / \omega \ll l$ and obtain an approximate solution given by

$$
h_{00} \approx q_{e} \frac{F_{e}}{w_{e}}\left(1-e^{i \sqrt{D}}\right)<H_{e}>
$$


with

$$
D=i \frac{\omega \epsilon \pi}{2 \bar{\nu} \hat{\omega}_{b}}\left(\frac{E}{T_{e}}\right)^{3 / 2} \frac{1}{Z_{e f f}+\Pi\left(E / T_{e}\right)}
$$

where $\xi=(.1-1+\epsilon) / 2 \epsilon$ is the normalized pitch angle variable and $\dot{\omega}_{b}$ is the normalized bounce frequency such that $\hat{\omega}_{b}=1$ at $\xi=1$. Plugging Eq. (62) into Eq. (24), we obtain the frequency shift due to the non-adiabatic response of trapped electrons with collision:

$$
\frac{\delta \omega}{\omega}=\frac{16 \pi^{3} \epsilon q_{e}^{2} v_{A}^{2} \sum_{l} \int \frac{E_{e}}{\omega_{b}} E d E d \xi\left(1-e^{i \sqrt{D} \xi}\right)\left(<H_{e}>_{l}\right)^{2}}{m_{e}^{2} c^{2} \omega^{2} R q T_{e} \int_{-\infty}^{+\infty} d \theta|\nabla S|^{2} \Phi^{2}}
$$

where subscrit $l$ denotes $/$ th trapped region in the infinite Fourier $\theta$ space. After evaluating $\left\langle H_{e}\right\rangle$ l using the analytic solution given by Eq. (62), summing over l, integrating over $\xi$ and $E$ and taking the imaginary part of $\delta \omega$. we finally obtain an explicit formula for the damping rate ie induced by trapped electrons:

$$
\frac{i_{e}}{\omega}=\frac{\sqrt{\pi / 8}}{1+\lambda^{2}}\left(I_{1}\left(\frac{s k_{\theta} \rho_{s}}{\Gamma}\right)^{2}+8 \lambda^{2} I_{2} q^{2} 3_{e}\right) \sqrt{\frac{\bar{\nu}}{\omega}}\left(\ln \left(16 \sqrt{\frac{\omega^{\prime} \epsilon}{\bar{\nu}}}\right)\right)^{-3 / 2}
$$

where $I_{1}$ and $I_{2}$ are two energy integration factors defined as follows:

$$
\begin{aligned}
& I_{1}=\int_{0}^{\infty} d z \frac{e^{-z}}{z^{1 / 4}} \sqrt{Z_{\text {ef } f}+\Pi(z)} \\
& I_{2}=\int_{0}^{\infty} d z \frac{e^{-z}}{z^{1 / 4}}(z-2)^{2} \sqrt{Z_{e f f}+\Pi(z)} .
\end{aligned}
$$

It is instructive to note that the first term in Eq. (6.5) comes from the parallel electrical field and is proportional to $\left(k_{a} p_{s}\right)^{2}$. whereas the second term comes from the curvature drift of trapped electrons and is independent of the mode number. This damping rate given by Eq. (65) is similar to that of Rosenbluth ${ }^{30}$ and also Gorelenkov and Sharapov. ${ }^{29}$ However, the logarithm scaling is different from that of Ref. 29. The difference comes from how the bounce average term $\left\langle H_{e}(\theta)\right\rangle$ is calculated. Our $-3 / 2$ scaling is obtained by noting that $\left.\left\langle H_{e}(\theta)\right\rangle \approx\left\langle H_{e}(\theta)-H_{e}(\pi)\right\rangle \gg H_{(-\pi}\right)$ for the TAE mode in the limit of $\xi \ll 1$.

\section{Application to the TFTR NBI Experiment}

Here we apply our formulation to the TFTR experiment by Wong et al. "Parameters for this experiment are $R=240 \mathrm{~cm}, a=75 \mathrm{~cm}, B=1.0 \mathrm{~T}$ and injected beam particle energy $E_{h}=110 k e q$. We consider the stability of the TAE modes at $r=30 \mathrm{~cm}$. 
where $q=1.32 . s=0.48, n_{e}=2.7 \times 10^{13} \mathrm{~cm}^{-3}, T_{e}=1.1 \mathrm{kev}, \beta_{e}=33_{i}=1.2 \%$. $Z_{\text {eff }}=2.5$, beam density scale length $L_{b}=18 \mathrm{~cm}$ and $z_{b} / v_{A}=1.1$. For the heam particle distribution, we take a slowing-down energy distribution with zero pitch angle. Thus, only circulating energetic particles are considered.

Figure 12 shows the critical beam beta values versus $k_{\theta} \rho_{b}$ for two values of $J_{p}$. The results are obtained by balancing the growth rate induced by beam particles with sum of ion Landau damping and collisional trapped electron damping. The continuum damping is assumed to be negligible. We find that the collisional trapped electron damping dominates over the ion Landau damping due to relatively small ion beta and large electron collisional frequency. The ion damping rate is calculated to be $\gamma_{i} / \omega=0.1 \%$. while the electron damping rate is $\gamma_{e} / \omega=\left[3.9\left(k_{\theta} \rho_{b}\right)^{2}+1.45\right] \%$ where we have converted $k_{\theta} \rho_{s}$ into $k_{\theta} \rho_{b}$, for $\Delta_{p}=0$. This collisional electron damping is much larger than the usual collisionless electron damping of $\gamma_{e} / \omega=q^{2} \beta_{e} v_{A} / v_{e}=0.2 \tau \%$. We note that the electron damping increases quadratically as a function of the mode number. On the other hand. the beam-induced growth rate increases initially for small $k_{\theta} \rho_{b}$, but saturates at finite value of $k_{\theta} \rho_{b}=1.0$. Thus, the critical beam beta increases as a function of mode number for finite value of kaph. In particular. for a toroidal mode number $n=2$ which corresponds to $k_{\theta} \rho_{b}=0.6$. the critical beta is $0.11 \%$. The effect of finite pressure gradient $\left(\Delta_{p}=0.0 T\right)$ increases the critical beta to $0.2 \%$. This range of the critical beta value is consistent with the experimental estimate of $3 . \approx 0.5 \%$.

\section{NON-LOCAL THEORY}

Here. we consider the energetic particle effects in the non-local limit and take into account the slow variation of the envelope function $A(r)$. We start from a quadratic form expressed in the real space. Equation (11) becomes. in the real $\theta$ space.

$$
\frac{\delta \omega}{\omega}=\frac{2 \pi \int J d \theta d \phi d t \int d^{3} v q_{j}\left(\hat{H}^{\sigma}\right)^{*} \hat{h}_{j}^{\sigma}}{\omega^{\prime} c^{2} \int J d \theta d \phi d t\left|\nabla_{\perp} \dot{\Phi}\right|^{2} / l^{2}}
$$

where denotes functions in the real space and $t=n q$ is the radial variable. Note that in the real space the integration domain in $\theta$ is $2 \pi$. Following Zonca and Chen. ${ }^{16}$ we write $\hat{\Phi}=\sum, \delta \dot{\Phi}_{j}(t) \exp (i n \phi-i j \theta)$ and assume the poloidal harmonics $\delta \dot{\Phi}_{j}(t)$ to have the form $\delta \hat{\Phi},(t)=A(t) \delta \dot{\Phi}(t, t-j)$, where $\dot{\Phi}(t, t-j)$ is a function of a fast variable $t-j$ and a slow variable $t$. while $A(t)$ is an envelope function of the slow variable only. In the spirit of ballooning representation, the fast varying function $\delta \dot{\Phi}(t . t-j)$ is written in terms of its Fourier transform

$$
\delta \hat{\Phi}(t, t-j)=\int_{-\infty}^{+\infty} d \theta^{\prime} e^{-i(t-j) \theta^{\prime}} \Phi\left(t . \theta^{\prime}\right)
$$


After summing over $j$, we obtain an alternative expression for $\hat{\Phi}$ given by

$$
\hat{\Phi}=\sum_{p} 2 \pi e^{i n \phi-i(\theta+2 \pi p)} A(t) \Phi(t, \theta+2 \pi p),
$$

where $p$ is an integer to be summed from $-\infty$ to $+\infty$. After plugging Eq. ( 70 ) into Eq. (68) and summing over $p$, we obtain a formula for the non-local growth rate $\hat{i}_{\alpha}$ induced by unergetic particles:

$$
\frac{\hat{\gamma}_{\alpha}}{\omega_{0}^{\prime}}=\frac{\int_{t 1}^{t 2}|A(t)|^{2} d t N_{\alpha}\left(t, \theta_{k}\right)}{\int_{t 1}^{t^{2}}|A(t)|^{2} d t W\left(t, \theta_{k}\right)}
$$

where $N_{\alpha}$ and $W$ are given in Eqs. (25) and (26). Note that in Eq. ( $i 1$ ) the integration domain in $\theta$ is infinite due to the summation of $p$, and $\theta_{k}$ is a function of $t$ to be determined. To solve $A(t)$, we express $A(t)$ in the eikonal form $A(t)=\exp \left(i \int \theta_{k} d t\right)$. Zonca and Chen ${ }^{16}$ found that the zeroth order $\theta_{k}$ satisfies the local dispersion relation as follows

$$
F\left(\tilde{\omega}, \theta_{k}, s, \Delta_{p}\right)=0
$$

whereas the next order of $\theta_{k}$ determines $A(t)$ to be

$$
A(t)=\frac{1}{\sqrt{\partial F / \partial \theta_{k}}} \sin \left(\int_{t_{1}}^{t} \theta_{k}\left(\bar{\omega} \cdot t^{\prime}\right) d t^{\prime}+\frac{\pi}{4}\right)
$$

where $t_{1}$ is one ot the two turning points of the local dispersion. It is instructive to make Eq. (j9) more transparent by exploiting the relation $W\left(t, \theta_{k}\right) \times \partial F / \partial \bar{\omega}^{2}$. Eq. (59) then becornes

$$
\hat{\hat{i}_{\alpha}} \approx \frac{1}{\pi} \int_{0}^{\pi} d \theta_{k} \hat{i \alpha}_{\alpha}\left(t \cdot \theta_{k}\right)
$$

where $\gamma_{\alpha}\left(t, \theta_{k}\right)$ is the local growth rate given by Eq. (24). Note that we have considered the $\sin ^{2}$ term in Eq. (71) has fast variation in $\theta_{k}$ as compared with that of $\gamma_{\alpha}\left(t, \theta_{k}\right)$ in the limit of $n \bar{\epsilon} \gg 1$, so that the $\sin ^{2}$ can be effectively replaced by $1 / 2$. From Eq. $(i t)$, we see that the non-local growth rate equals the local growth rate averaged over $\theta_{k}$. Numerically, we find that the non-local growth rate induced by fusion alpha particles is smaller than the local growth rate evaluated at $\theta_{k}=0$.

\section{DISCUSSIONS AND CONCLUSIONS}

We have developed a pert urbative formulation for the stability of high-n TAE mode in the presence of Alfvénic energetic particles and fusion alpha particles in totamak 
plasmas. Our formulation includes the destabilizing effects of energetic particles and stabilizing effects of thermal electron collisional damping and thermal ion Landau damping. The continuum damping can also be self-consistently included by taking into account the two dimensional wave structure of the TAE mode. For energetic particles, full finite Larmor radius effects and main drift orbit width effects are retained by employing gyro-kinetic equation, whereas for thermal ions, the lowest order FLR terms are kept in the calculation of the parallel electrical field that is important for the collisional electron damping. The continuum damping can also be self-consistently included by taking into account the two dimensional wave structure of the TAE mode.

We have studied extensively the parameter dependence of the growth rate of the TAE mode induced by fusion alpha particles in the local limit (i.e, in the limit of translational invariance in the ballooning representation). In this local limit, the growth rate induced by circulating alpha particles is found to increase linearly with the toroidal mode number $n$ for small $k_{\theta} \rho_{\alpha} \ll 1$, and decrease as $1 / n$ for large $k_{\theta} p_{\alpha} \gg 1$. The maximum growth rate occurs at $k_{\theta} \rho_{\alpha}$ on an order of unity and is nearly constant for the range of $0.7<v_{a} / v_{A}<2.5$. The value of the maximum growth rate due to the circulating alpha particles is approximately given by $\left(\gamma_{\alpha} / \omega^{\prime}\right)_{\max } \approx-3 q^{2} r \cdot 3_{x}^{\prime}$. where the prime denotes the derivative with respect to the plasma radius $r$. On the other hand. the trapped alpha particle contribution to the growth rate is dominated by the precessional drift resonance. The bounce resonance contribution is negligible. The growth rate induced by the trapped alpha particles peaks sharply at $k_{\theta} \rho_{0} \approx$ $v_{A} / q v_{\alpha}$ where the precessional drift resonance occurs for the most energetic particles. The maximum growth rate due to the trapped particles is given by $\left(\gamma_{\alpha} / \omega^{\prime}\right)_{\max } \approx$ $\sqrt{2 \epsilon} \pi^{2} q^{2} r 3_{\alpha}^{\prime} J_{0}^{2}\left(v_{A} / q v_{\alpha}\right)$.

The effects of the finite plasma beta are considered. We find that the alpha particle destabilizing contribution is reduced by the effects of finite pressure gradient. In particular, the growth rate induced by alpha particles vanishes as the pressure gradient parameter $\Delta_{p}$ approaches the critical value $\left(\Delta_{p}\right)_{\text {crit }}$. where the TAE morle begins to merge into the Alfvén continuum.

The global wave structure is taken into account. In the global theory, the growth rate induced by alpha particles equals the $\theta_{k}$-averaged local growth rate and is usually smaller than the local growth rate evaluated at $\theta_{k}=0$. Furthermore. the continuum damping can be included in the global theory.

In order to drive the TAE modes unstable. the destabilizing contribution of energetic particles must overcome the thermal ion Landau damping, the electron collisional damping and the continuum damping. As an example. we consider the critical alpha beta for ITER ${ }^{31}$ parameters, taking all the damping mechanisms into account. We take the parameters of $B=4.85 T . R=600 \mathrm{~cm}$, and $a=21.5 \mathrm{~cm}$ and consider following 
local values: $r=60 \mathrm{~cm}, q=1.0, s=0.6, n_{e}=10^{14} \mathrm{~cm}^{-3}, T_{e}=10 \mathrm{kev}, T_{i}=10 \mathrm{kve}$, $Z_{\text {eff }}=1.0$, and the alpha particle density profile is $n_{\alpha}(r)=n_{\alpha}(0) \exp \left[-\left(r / L_{\alpha}\right)^{2}\right]$ with the scale length $L_{\alpha}=90 \mathrm{~cm}$. Using Eq. (56), we find the ion Landau damping due to deuteriums and tritiums is $\gamma_{i} / \omega=0.71 \%$. The electron collisional damping is evaluated using Eq. (65) and is $\gamma_{e} / \omega(\%)=0.33\left(k_{\theta} \rho_{\alpha}\right)^{2}+0.23$, where we have assumed zero pressure gradient. In the local limit, we find the critical alpha beta for excitation of the high-n TAE mode is $\beta_{\alpha c}(0)=0.5 \%$, and the most probable mode number to be excited is $n \approx 6$. In the non-local theory, the critical beta value is increased to $\beta_{\alpha c}(0)=1.0 \%$ for the local parameters used here. The critical beta value wil be further increased due to the finite pressure gradient effect and the continuum damping.

In conclusion, we have presented a comprehensive formulation for the stability of high-n TAE modes by taking into account the destabilizing effects of energetic particles or fusion alpha particles and the stabilizing effects of thermal ion Landau damping, electron collisional damping and the continuum damping. The alpha particle contribution to the growth rate as a function of $k_{\theta} \rho_{\alpha}$ has a maximum at $k_{\theta} \rho_{\alpha}$ on an order of unity. For ITER-like parameters, the critical alpha beta $3_{\alpha c}(0)$ is on an order of $1 \%$. The detailed parameter studies of the TAE stability for the planned TFTR D-T experiment ${ }^{32}$ and for the ITER will be the subject of a future paper.

\section{ACKNOWLEDGMENT}

We acknowledge useful discussions with L. Chen, H. L. Berk, H. Biglari. J. W. Van Dam. K. L. Wong and F. Zonca. This work was started at Centre de Recherches en Physique des Plasmas in Lausanne. Switzerland when one of the authors ( $\mathrm{C}$. Y. Fu) was working there. It is supported by the Fonds National Suisse pour la Recherche Scientifique, and by the $I$. S. Department of Energy Contract No. DE-AC02-76('HO-3073. 


\section{TREERENCES}

${ }^{1}$ M. N. Rosenbluth and P. H. Rutherford, Phys. Rev. Lett. 34, 1428(1975).

${ }^{2}$ A. B. Mikhailovskii, Sov. Phys. JETP 41, 980 (1975).

${ }^{3}$ C. Z Cheng, L. Chen, and M. S. Chance, Ann. Phys. (N. Y.) 161, 21 (1984).

${ }^{4}$ C. Z. Cheng and.M. S. Chance, Phys. Fluids 29, 3695 (1986).

${ }^{5}$ G. Y. Fu, Ph. D. Thesis, The University of Texas at Austin (1988); Institute for Fusion Studies Report. No. IFSR-325 (1988).

${ }^{6}$ G. Y. Fu and J. W. Van Dam, Phys. Fluids B1, 1949 (1989).

'C. Z. Cheng, G. Y. Fu, and J. W. Van Dam, in Theory of Fusion Plasmas 1988. ed. J. Vaclavik, F. Troyon. and E. Sindoni (Societa Italiana di Fisica/Editrice Compositori, Bologna, 1989), p. 259.

${ }^{8}$ L. Chen. ibid., p.327.

${ }^{9}$ J. IV. Van Dam, C. Y. Fu, and C. Z. Cheng, Fusion Technology 18, 461 (1990).

${ }^{10} \mathrm{C}$. Z. Cheng, Phys. Fluids, B3, 2.t63 (1991).

${ }^{11}$ K. L. Wong, R. J. Fonk, S. F. Paul, D. R. Roberts. E. D. Fredrickson, R. Nazikian. H. K. Park. M. Bell. N. L. Bretz, R. Budny, S. Cohen. G. W. Hammett. F. C'. Jobes, D. M. Meade, S. S. Medley, D. Mueller, Y. Nagayama, D. K. Owens. and E. J. Synakowski, Phys. Rev. Lett. 66, 1874 (1991).

${ }^{12}$ W. W. Heidbrink, E. J. Strait, E. Doyle, and R. Snide, Nucl. Fusion 31. 1635 (1991).

${ }^{13}$ H. L. Berk. J. W. Van Dam. Z. Guo, and D. W. Lindberg, to appear in Phys. Fluids B.

${ }^{14} \mathrm{~F}$. Zonca and L. Chen, Phys. Rev. Lett., 68, 592(1992)

${ }^{15}$.M. N. Rosenbluth, H. L. Berk, D. M. Lindberg, and J. W. Van Dam, Phys. Rev. Lett., 68, 596 (1992).

${ }^{16} \mathrm{~F}$. Zonca and L. Chen, to be submitted to Phys. Fluids, 1992. 
${ }^{17}$ L. Villard and G. Y. Fu, submitted to Nucl. Fusion, 1992.

${ }^{18}$ H. Biglari, L. Chen, and F. Zonca, Rep. PPPL-2789, Plasma Physics Laboratory, Princeton U'niversity, Princeton, NJ (1991); unpublished.

${ }^{19}$ D. A. Spong. J. A. Holmes, J. - N. Leboeuf, and P. J. Chritenson. Fusion Technology 18, $496(1990)$.

${ }^{20}$ G. Rewoldt, Nucl. Fusion 31, 2333 (1991).

${ }^{21}$ H. L. Berk, B. N. Breizman, and H. Ye, Phys. Lett. A162, 475 (1992).

${ }^{22}$ R. Betti and J. P. Freidberg, Phys. Fluids B3, 1865 (1991).

${ }^{23}$ T. . M. Antonsen and B. Lane, Phys. Fluids 23, 1205 (1980).

${ }^{24}$ P. J. Catto, W. M. Tang, and D. E. Baldwin, Plasma Physics 23, 639 (1981).

${ }^{25}$ M. N. Rosenbluth, D. W. Ross, and D. P. Kostomarov, Nucl. Fusion 12, 3 (1972).

${ }^{26}$ X. Q. Xu and .V. N. Rosenbluth, Phys. Fluids B 3. 363 (1991).

${ }^{27}$ J. W. Connor. R. J. Hastie, and J. W. Taylor, Phys. Rev. Lett. 40, 396 (1978).

${ }^{28}$ G. Y. Fu and C. Z. Cheng, Phys. Fluids B 2, 985 (1930).

${ }^{29}$ N. N. Ciorelnkov and S. E. Sharapov, Physica Scripta. t:; (1991).

${ }^{30}$ M. N. Rosenbluth, private communication.

${ }^{31}$ D. E. Post. K. Borrass, J. D. Callen. S. A. Cohen, J. G. Gordey. F. Engelmann, N. Fujisawa, .H. F. A. Harrison, J. T. Hogan. H. J. Hopman, Y. Igithanov, O. Kardaun. S. M. Kaye S. Krasheninnikov. A. Kukushkin. V. Mukhovatov, W. M. Nevins. A. Vocentini. G. W. Pacher, H. D. Pacher, V. V. Parail. L. D. Pearlstein. L. J. Perkins. S. Putvinskij. K. Riedel, D. J. Sigma, M. Sugihara. D. IV. Swain. T. Takizuka. K. Tani, T. Tsunematsu, N. A. Uckan, J. G. Wegrowe. J. Wesley, S. Yamamoto. R. Yoshino, $K$. Young, P. N. Yushmanov, and international contributors. ITER Documentation Series. No. 21 (IAEA, Vienna, 1991).

${ }^{32}$ R. V. Budny et al., Nucl. Fusion 32, 429 (1992). 


\section{FIGURE CAPTIONS}

Figure 1. Growth rates induced by circulating alpha particles as a function of $v_{\alpha} / v_{A}$ for $s=0.6, \Delta_{p}=0$ and $k_{\theta} \rho_{\alpha}=1.0$.

Figure 2. Function $H(k)$ for $s=0.6, \Delta_{p}=0, v_{\alpha} / v_{A}=1.0$ and $k_{\theta} \rho_{\alpha}=1.0$.

Figure 3. Growth rate induced by circulating alpha particles as a function of $k_{\theta} \rho_{\alpha}$ for $s=0.6, \Delta_{p}=0$ and $v_{\alpha} / v_{A}=2.0$.

Figure 4. The maximized growth rate induced by circulating alpha particles and the corresponding $k_{\theta} \rho_{\alpha}$ as a function of $v_{\alpha} / v_{A}$ for $s=0.6$ and $\Delta_{p}=0$.

Figu'e 5 . The maximized growth rate induced by circulating alpha particles and the corresponding $k_{\theta} \rho_{\alpha}$ as a function of magnetic shear is for $\Delta_{p}=0.0$ and $u_{\alpha} / u_{A}=2$.

Figure 6. The maximized growth rate induced by circulating alpha particles and the corresponding $k_{\theta} \rho_{\alpha}$ as a function of the pressure gradient parameter $\lambda_{p}$ for $s=0.6$ and $v_{\alpha} / v_{A}=2$.

Figure 7 . Growth rates induced by trapped alpha particles as a function of $r_{\alpha} / v_{t}$ for $s=0.6, \Delta_{p}=0$ and $k_{\theta} \rho_{\alpha}=1.0$.

Figure 8. Growth rate induced by trapped alpha particles as a function of $k_{n} \rho_{x}$ for $s=0.6 . \Delta_{p}=0$ and $u_{\alpha} / v_{\alpha}=2.0$.

Figure 9. The maximized growth rate induced by trapped alpha particles and the corresponding $k_{\theta} \rho_{\alpha}$ as a function of $v_{\alpha} / v_{A}$ for $s=0.6$ and $\Delta_{p}=0$.

Figure 10. The maximized growth rate induced by trapped alpha particles and the corresponding $k_{\theta} \rho_{\alpha}$ as a function of magnetic shear $s$ for $\Delta_{p}=0.0$ and $\nu_{\alpha} / \mu_{1}=2$.

Figure 11. The maximized growth rate induced by trapped alpha particles and the corresponding $k_{\theta} \rho_{\alpha}$ as a function of the pressure gradient parameter $\Delta_{p}$ for $s=0.6$ and $v_{\alpha} / v_{A}=2$.

Figure 12. The critical beam beta value versus $k_{\theta} \rho_{b}$ for two values of $\Delta_{p}$ at $r=30 \mathrm{~cm}$ for the TFTR TAE experiment. 


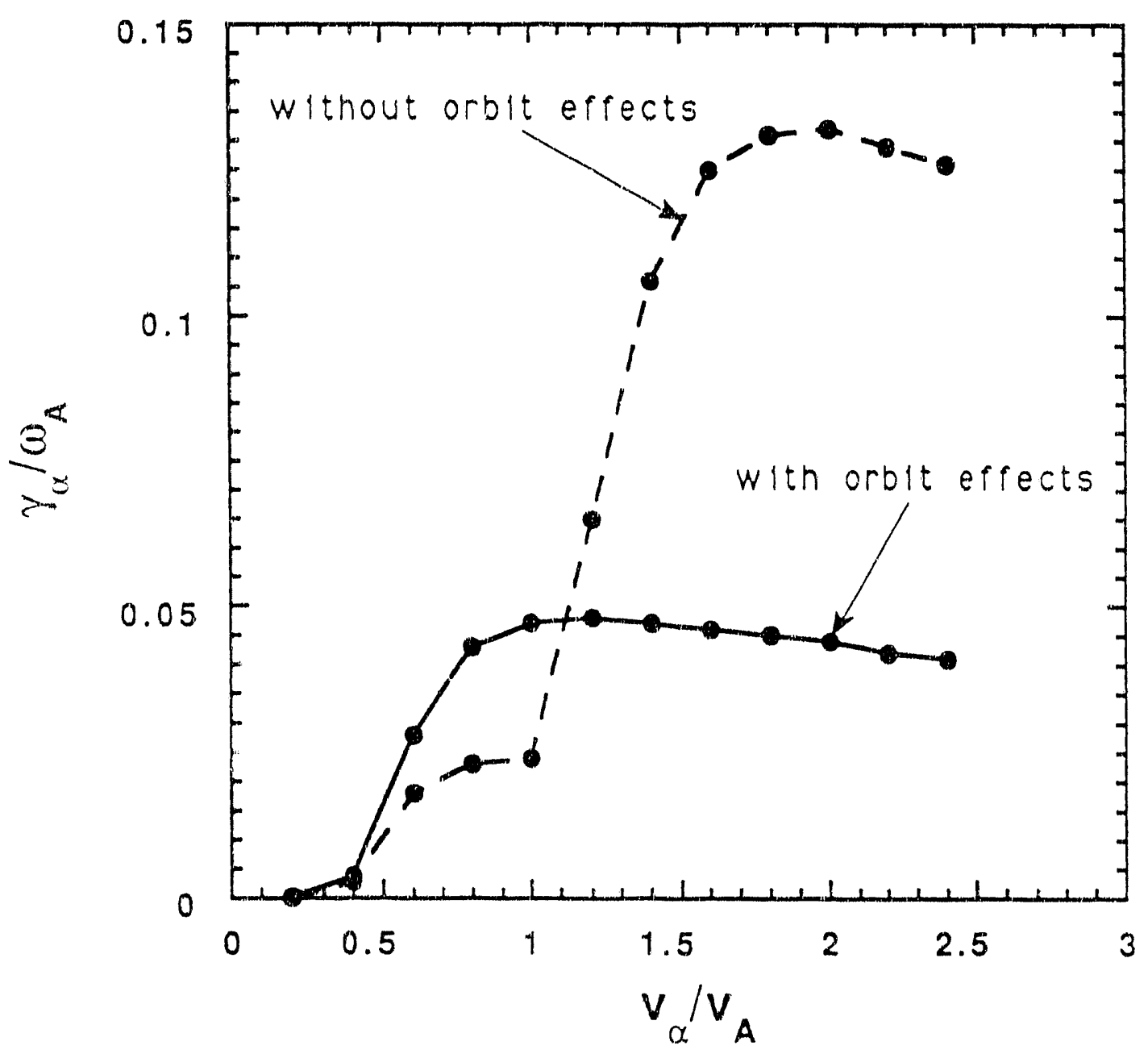

Fig. 1 


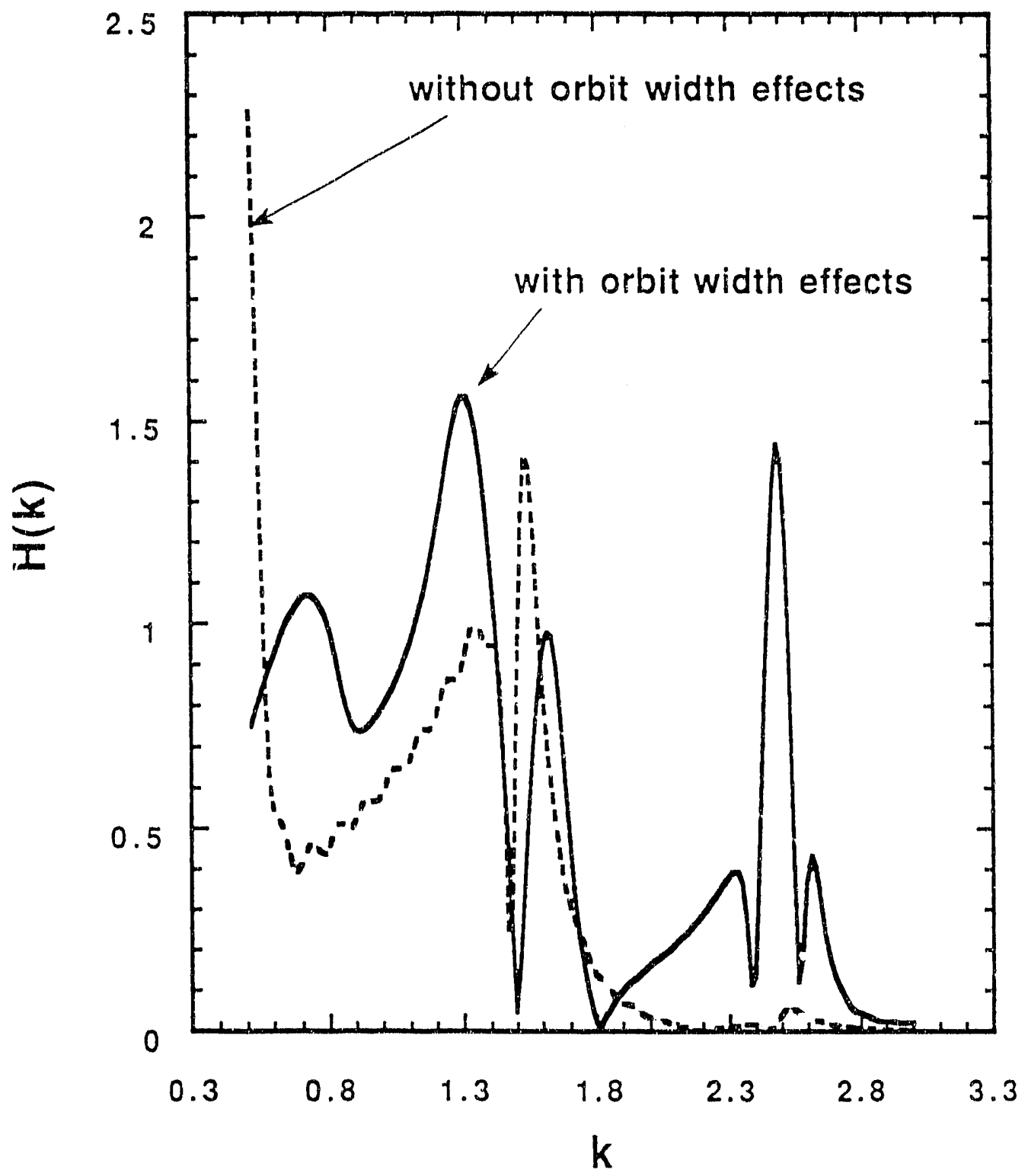

Eig. 2 


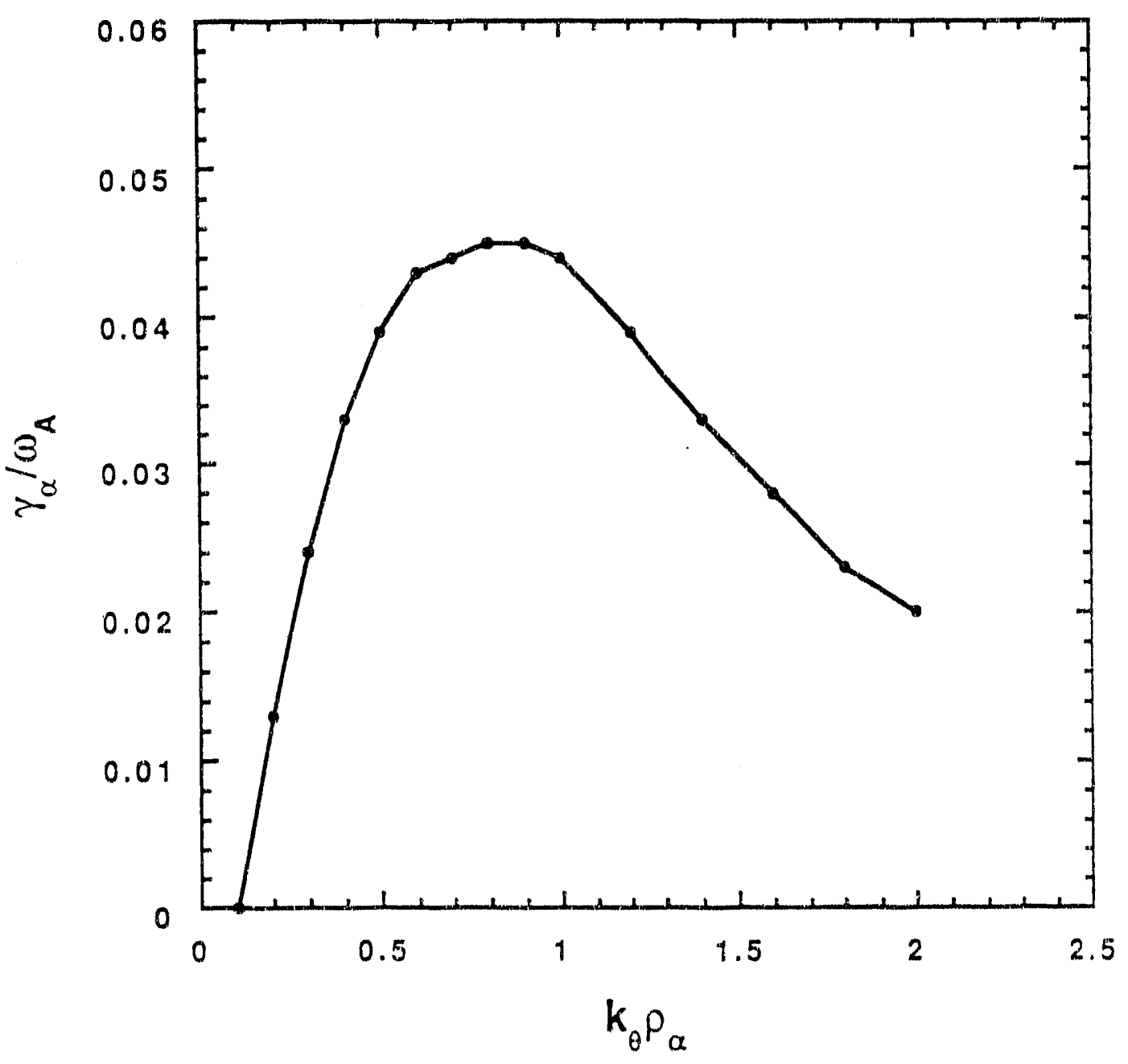

Fig. 3 


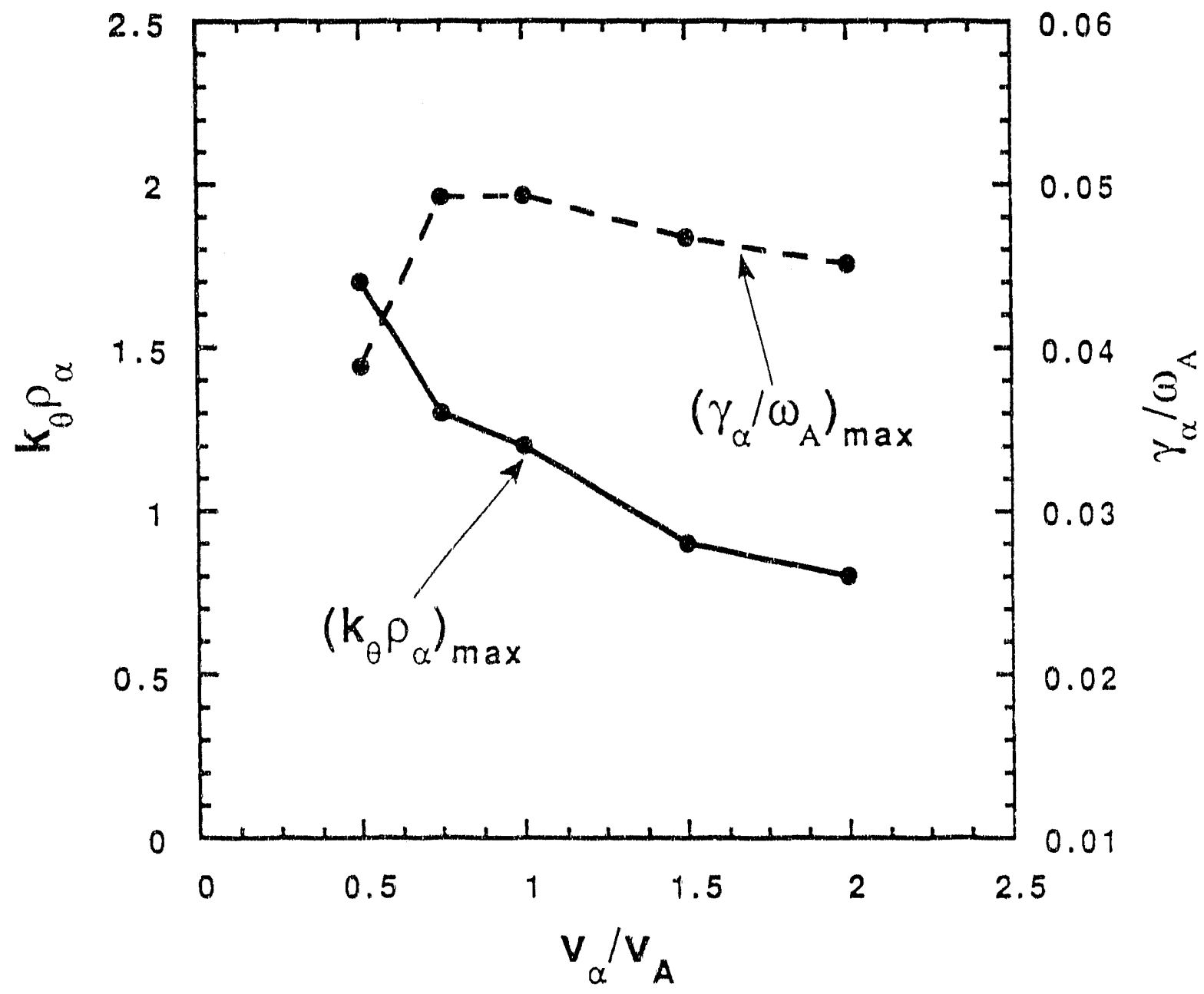

Fig. 4 


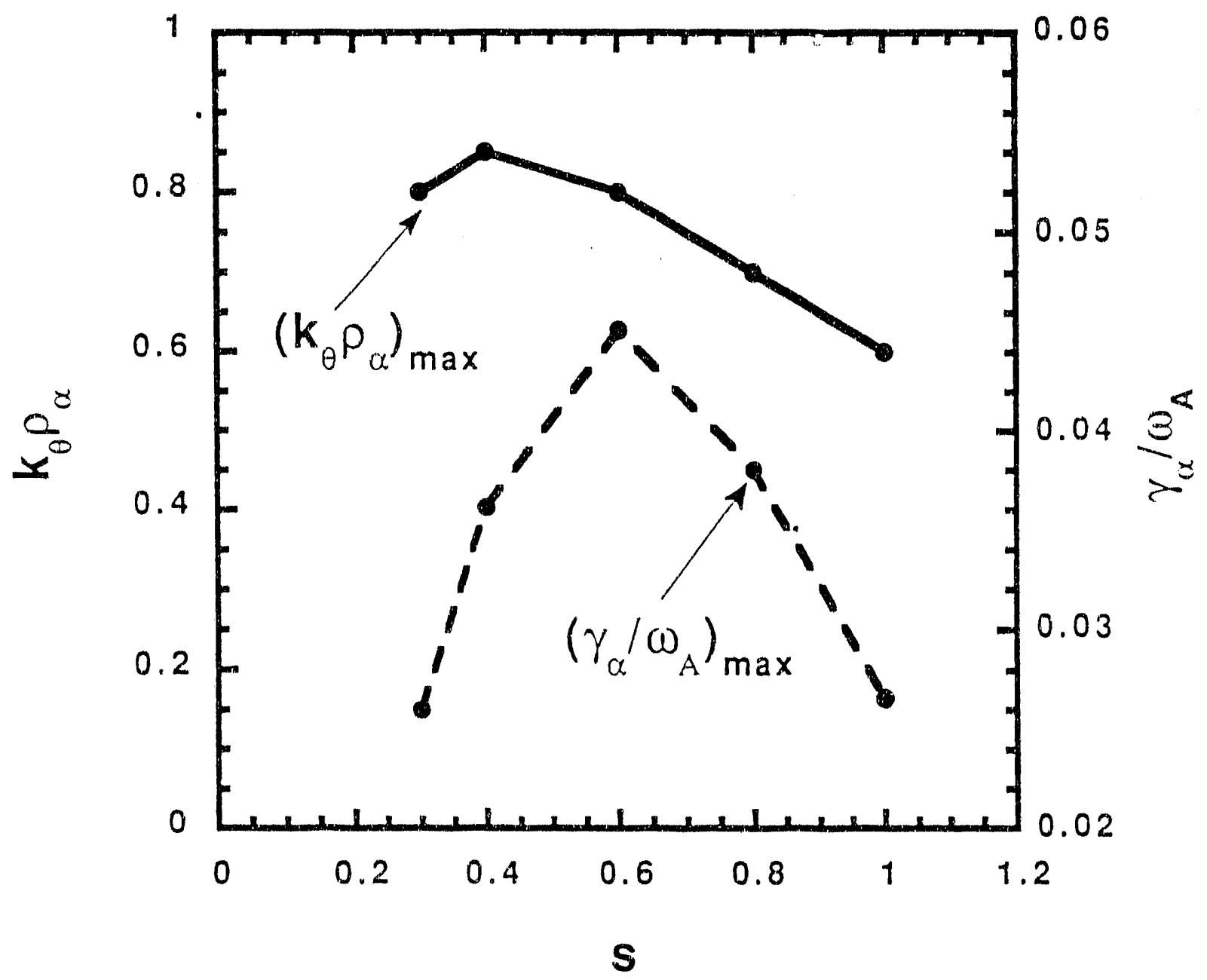

Fig. 5 


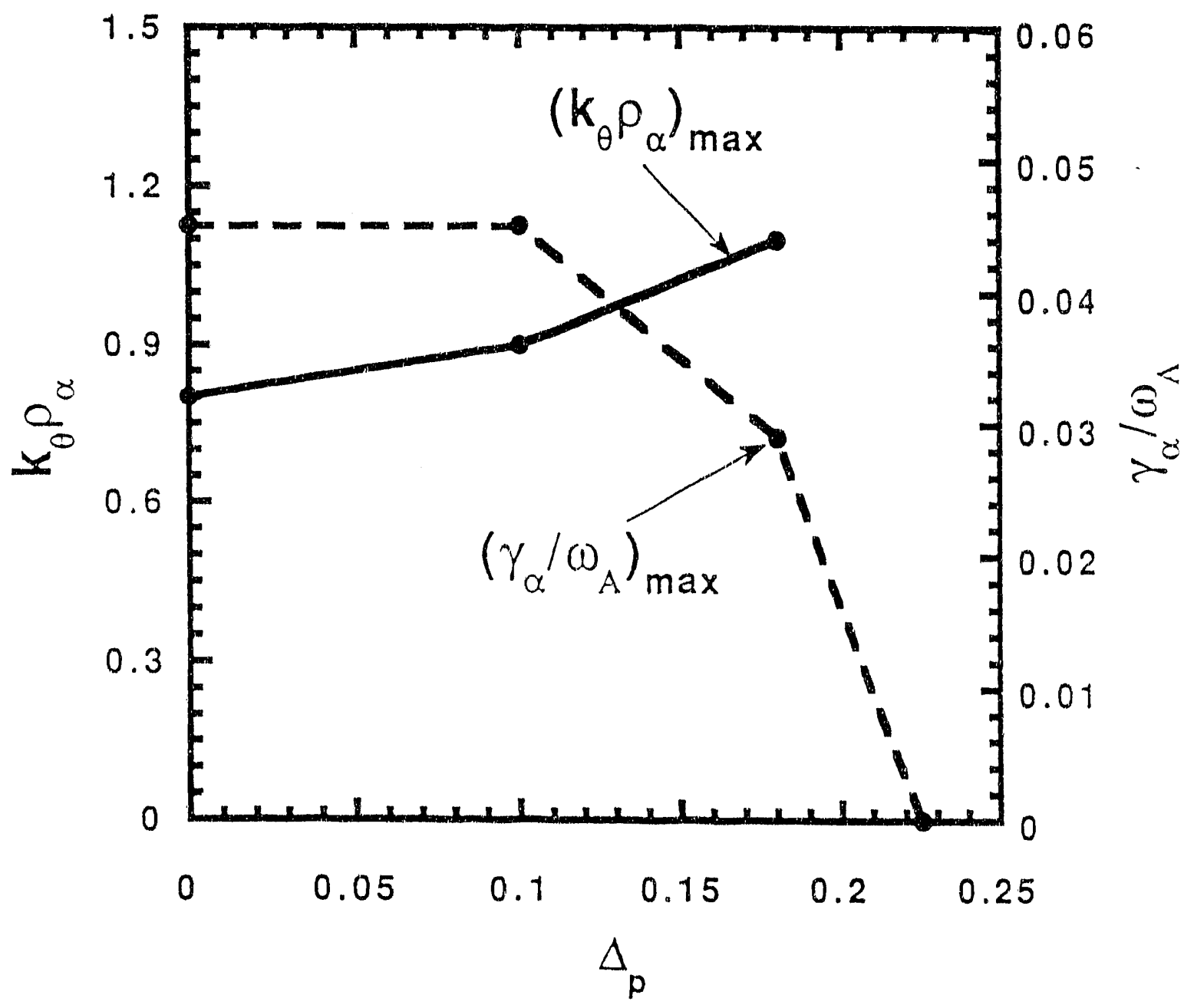

Fig. 6 


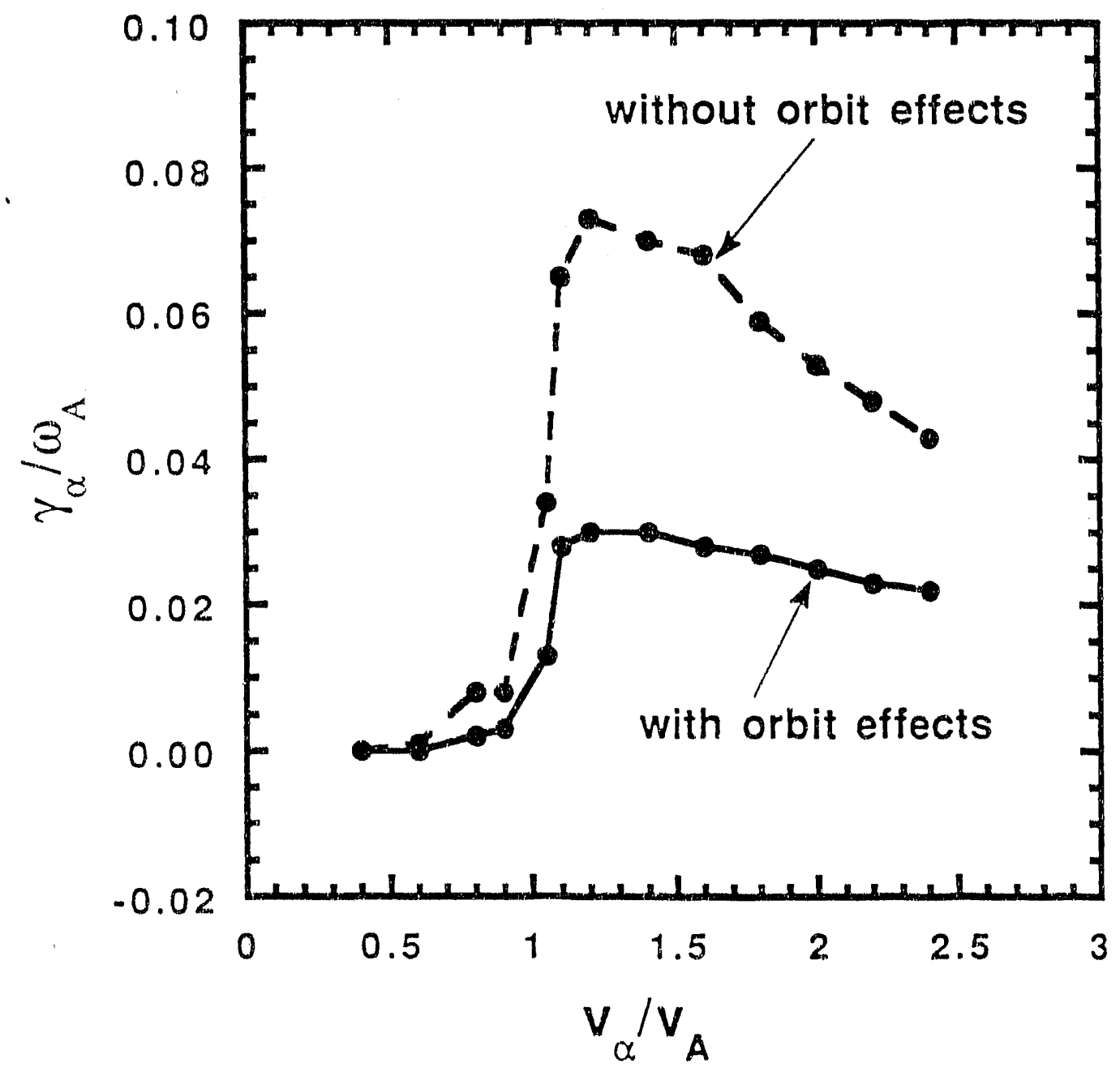

Fig. 7 


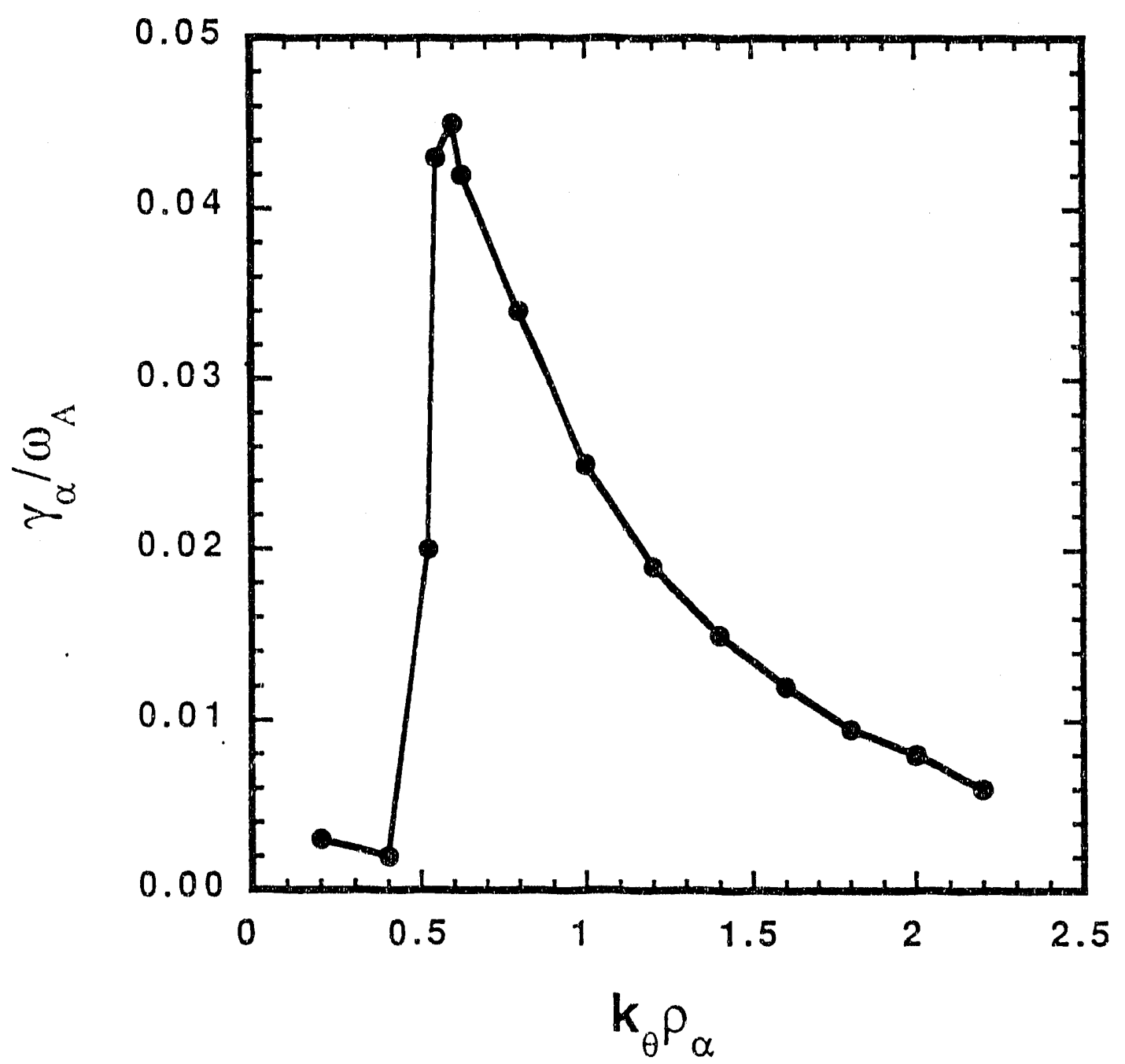

Fig. 8 


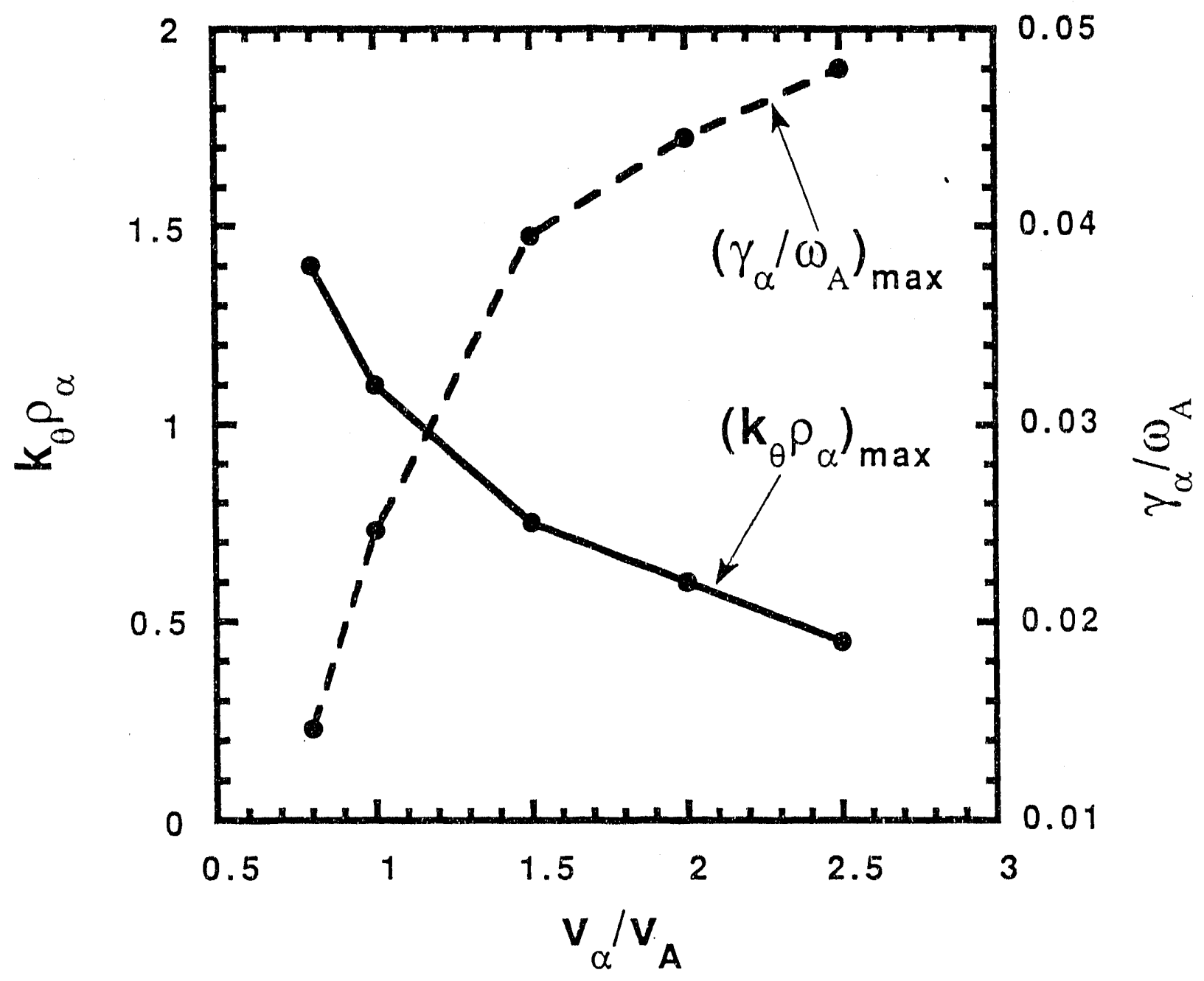

Fig. 9 


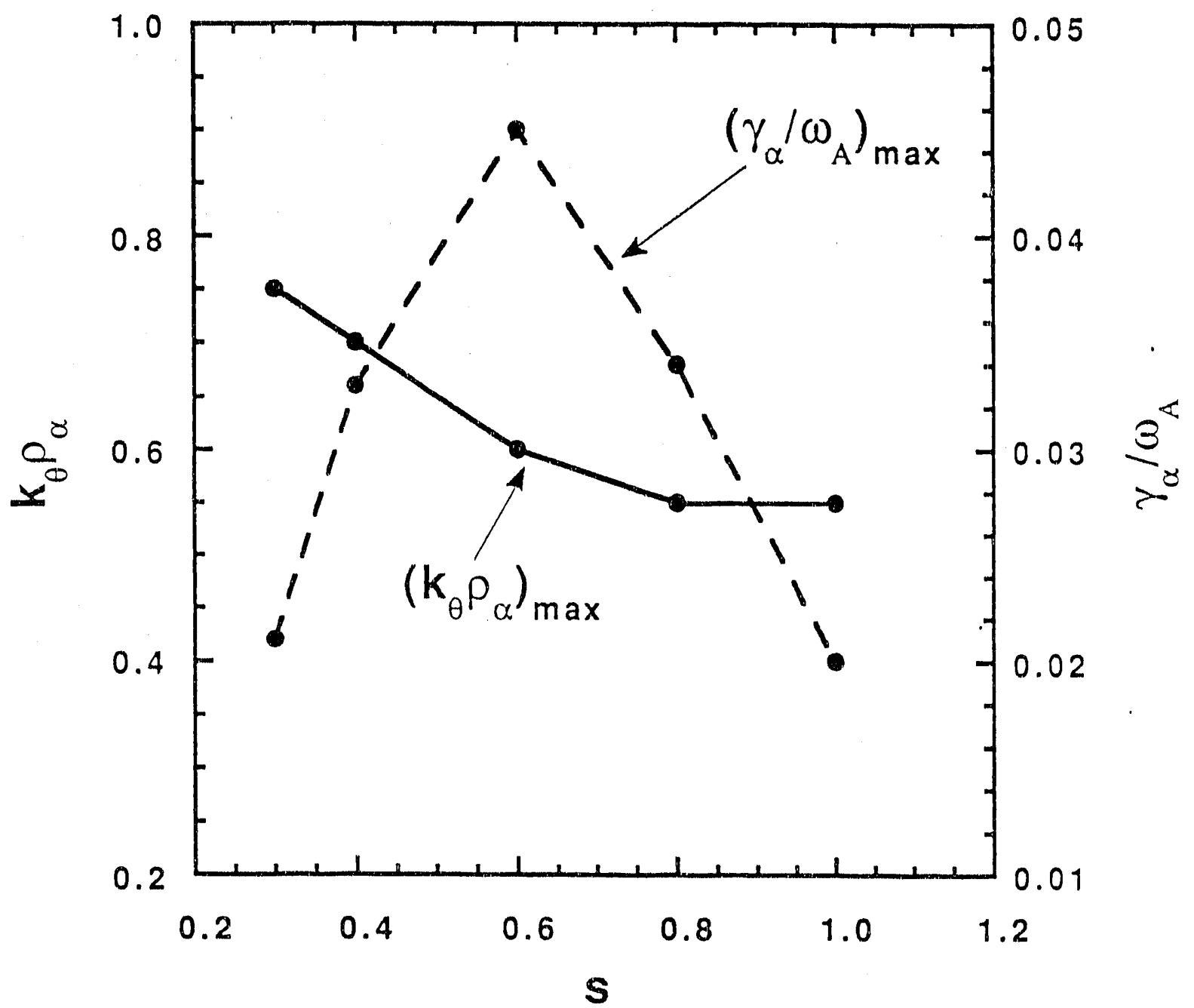

Fig. 10 


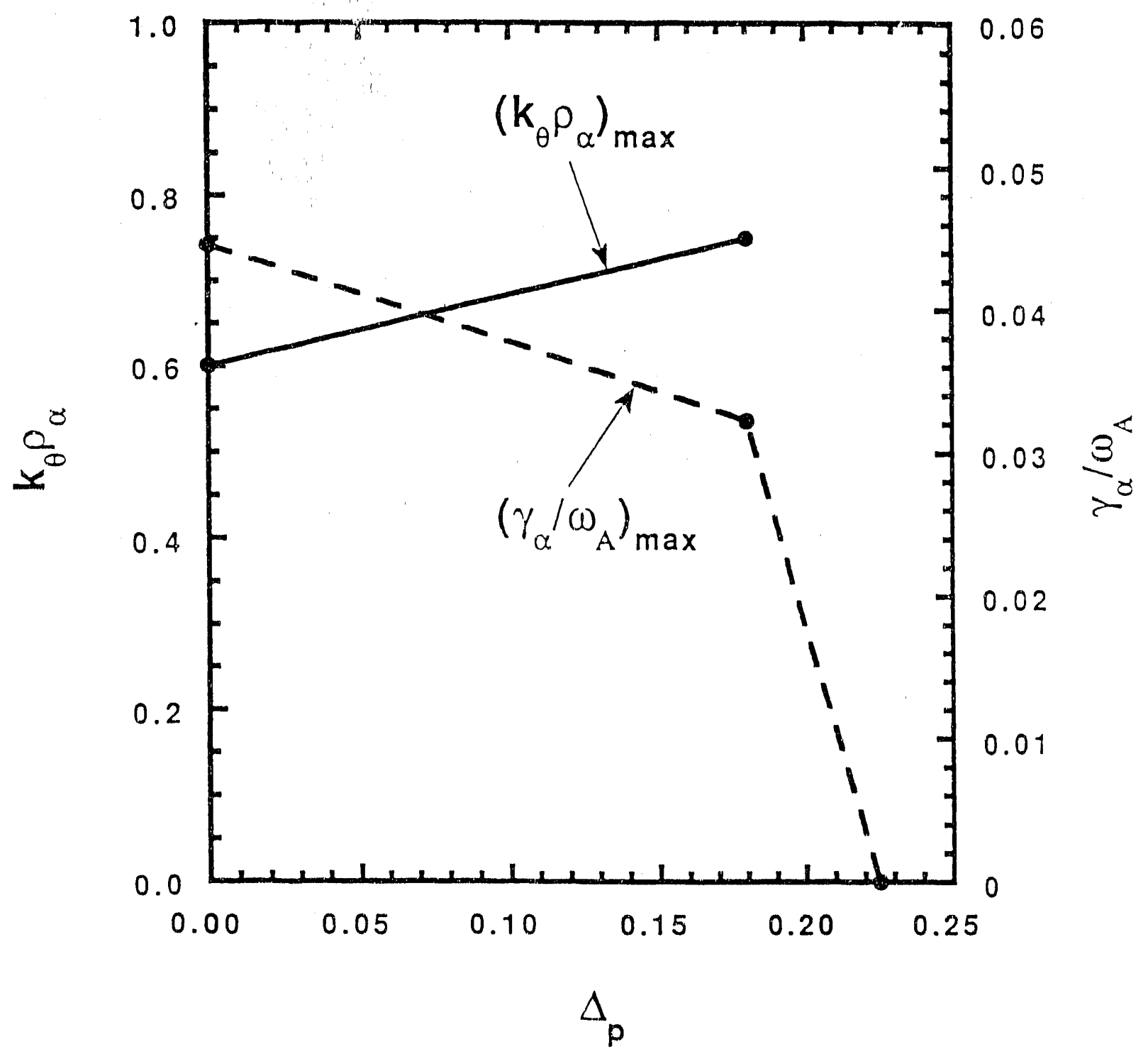

Fig. 11 


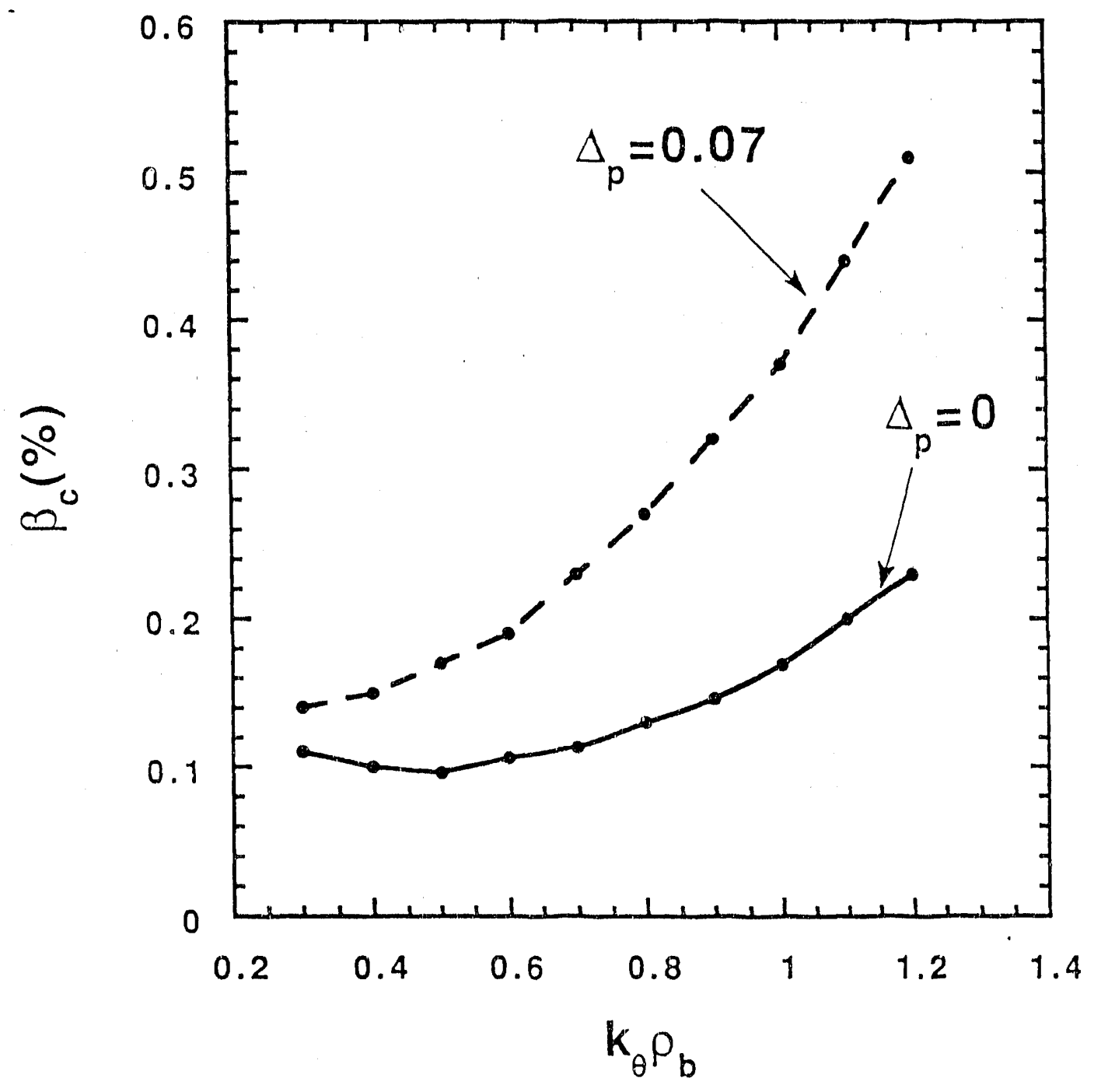

Fig. 12 
Dr. F. Peolonv, Univ, of Wothongong, AUSTRALIA

Prof. M.H. Brennan, Univ. of Syonoy, AL'STRALIA

Plasma Research Lab., Austrelyay Nat Univ. AUISTRALIA

Prof. I.A. Jones, Flinders Univ, AUSTRALIA

Prof. F. Cap, Inst. for Theoretical Physics, AUSTRIA

Prot. M. Heindier, Institut for Theoretisctio Physik, AUSTRIA

Prot. M. Goossone. Astronomisch InstituUt, BELGIUM

Ecole Royade Mitivire, Lob. do Phy. Plaemas, BELGIUM

Commission-ELropeen, DG. XII-Fusion Proo., BELGIUM

Prof. R. Bouciqut, Rijkeuniverziton Gent, BELGIUM

Dr. P.H. Sakanaka, Inssituro Frica, BRUZIL.

Inettuto Neciond Do Porquizas Especieir-INPE, BRUZIL

Documents Orico, Abomic Enorgy of Cmade Ld., CANADA

Dr. M.P. Bectryneki, MPB Tecthologies, Inc., CANADA

Dr. H.M. Skaregard, Univ. of Sackatctiowen, CANADA

Prof. J. Toictumam, Univ. of Moritread, CANADA

Prot. S.R. Sroenivasen, Univ. of Calgary, CANADA

Prof. T.W. Jetunston, INAS-Energio, CANADA

Dr. R. Botron, Contro canadion de tusion meanstique, CANADA

Dr. C.R. James, Univ. \& Nborta, CANADA

Dr. P. Lukte, Komenekeno Univorsziti, CZECHO-SLOVAKIA

The Librarian, Cutham Leboratory, ENGLAND

Libran, R61, Ruthertord Apploton Laboratory, ENGLAND

Mrs. S.A. Hutchinaon, JET Libray, ENGLAND

Dr. S.C. Sharma UnN. of South Pacific. FIJI ISLANDS

P. Manonen, Univ. of Helsinki, FINLAND

Prof. M.N. Buseac, Ecolo Polyochnious, FRANCE

C. Moutied, Lab. Physique des Milioux ionisos, FRANCE

J. Radot, CEN/CADARACHE - Bat 506, FRANCE

Prot. E. Economow, Univ. of Crote, GREECE

Ms. C. Pinni, Univ. of loannina, GREECE

Dr. T. Mud, Academy Bibliographic Ser., HONG KONG

Proprint Library, Hungarian Acadomy of Sci., HUNGARY

Dr. B. DasGupta, Saha inst. of Nuclear Ptysica, INDIA

Dr. P. Kaw, Inet. for Plasm Rosearch, INDIA

Dr. P. Rocenew, lereed inst of Tecthnology, ISRAEL

Librarian, Intemationa contor for Thoo Physica, ITALY

Mizis C. Do Palo, Associazion EURATOMENEA, ITALY

Dr, G. Groseo, lstituro de Fisica ded Plasma, ITALY

Prot. G. Rostangoni, lestuto Gas konizzati Dod Cnr, ITALY

Dr. H. Yomato, Toshiba Ros a Devel Center, JAPAN
Prot. 1. Kowakemi, Hiroshima Univ., JAPAN

Prof. K. Nishikawa, Hroshima UnN., JAPAN

Drector, Jepen Alomic Energy, Reseanch Inst, JAPAN

Prof. S. Itoh, Kyushu Univ., JAPAN

Posearch into. Ctr., National Insot. for Fusion Scrence, JAPAN

Prof. S. Tanaka, Kyoto Univ., JAPAN

Librey, Kyoto Univ., JAPAN

Prof. N. Inow, Univ. of Tokyo, LAPAN

Secrotery, Pleama Section, Eloctrobchnical Lab., JAPAN

S. Mori, Tectmical Advisor, LAERI, LAPAN

Or. O. Mitarai, Kumemola Inst of Technology, JAPAN

J. Hyeon Sook, Korea Atomic Enorgy Roseanch inst, KOREA

D.1. Chai, The Korea Adv. Inst of Sa. \& Tech., KOREA

Prot. B.S. Liloy, Univ. of Wrikato, NEW ZEALAND

Inat of Phyrias, Chinese Acad Sa PEOPLE'S REP OF CHINA

Libren, Inst of Plasma Physics, PEOPLE'S REP OF CHINA

Tringhua Univ. Lbrary, PEOPLE'S REPUBLIC OF CHINA

2. L. S.W. Inst Physica, PEOPLE'S REPUBUC OF CHINA

Prof. J.A.C. Cabred, Instituto Superior Teconico, PORTUGAL

Dr. O. Potrus, AL I CUzA Univ., ROAMANIA

Dr. J. Villiers, Fusion Studios, AEC, S. AFRICA

Prot. M.A. Hellberg, Univ. of Matal, S. AFRICA

Prol. D.E. Km, Poheng Inst. of Sa. \& Tech., SO. KOREA

Prof. C.I.E.M.A.T, Fusion Division Library, SPAIN

Dr. L Stonto, Univ. of UMEA, SWEDEN

Libray, Royd inst. of Tectnology, SWEDEN

Prot. H. Wilhetmeon, Chesmers Univ. of Tech., SWEDEN

Contro Phys. Dos Plasmas, Ecolo Polyrech, SWITZEALAND Bibliothook, Inst. Voor Plasma-Fysica, THE NETHERLANDS

Asst. Prot. D. S. Colkr, East Toch. UnN. TURKEY

Dr. V.A. Gukhikh, Sa. Ros. Inst Eloctrophys.I Apparatus, USSR

D. D.D. Ayubr, Siberian Brench of Academy of Sa., USSR

D. G.A. Elicoov, I.Y. Kurchatov Inst, USSR

Librarien. The Ukr. SSP Acadomy of Scionces, USSR

Dr. LM. Kowrizhnykh, Inst. of Genora Physics. USSR

Komtorschungsaniege GmbH, Zentralbibliotheix, W. GERMANY

Bibliothok, Inst. For Plasmatorechung. W. GERMANY

Prot. K. Schinder, Ruhr.Uniwer sitht Bochium, W. GERMANY

Dr. F. Wegner, (ASOEX), Max-Planck-Institut, W. GERMANY

Librenien, Max-Plenck-Institur, W. GERMANY

Prot. R.K. Janeer, Inst of Prysica, YUGOSLAVIA 
